Supporting Information:

\title{
Electrocatalytic Hydrogen Evolution at Low Overpotentials by Cobalt Macrocyclic Glyoxime and Tetra-Imine Complexes
}

\author{
Xile Hu, Bruce S. Brunschwig, and Jonas C. Peters* \\ Division of Chemistry and Chemical Engineering, Arnold and Mabel Beckman \\ Laboratories of Chemical Synthesis, California Institute of Technology, Pasadena, \\ California 91125. Fax: 01626 5774088; Tel: 01626 3954036; Email: \\ jpeters@caltech.edu.
}




\section{General Parameters for Simulation}

\begin{tabular}{|c|c|c|c|c|c|c|}
\hline Scan Rate & Cycles & $\mathrm{Ru}(\mathrm{Ohm})$ & $\mathrm{CdI}(\mathrm{F})$ & Temp.(K) & Geometry & Area $\left(\mathrm{cm}^{2}\right)$ \\
\hline $0.1 \mathrm{~V} / \mathrm{s}$ & 1 & 0 & 0 & 298.2 & Planar & 0.07 \\
\hline
\end{tabular}

\begin{tabular}{|c|c|c|c|}
\hline Diffusion & Potential Steps (V) & Gauss-Newton Iteration & Noise Level (A) \\
\hline Semi-Infinite 1D & 0.005 & 2 & 0 \\
\hline
\end{tabular}

Space perpendicular to electrode:

\begin{tabular}{|c|c|c|}
\hline Grid Expansion Factor & Rel. Truncation Error & Xmas/SQRT(Dt) \\
\hline 0.5 & 0.0001 & 6 \\
\hline
\end{tabular}

Local FEM error level: 0.05 
Steady State analysis of the bimetallic pathway

We have written the kinetic equations for the bimetallic pathway as:

$$
\begin{gathered}
\mathrm{Co}(\mathrm{I})+\mathrm{HA} \rightarrow \mathrm{Co}(\mathrm{III})-\mathrm{H}+\mathrm{A}^{-} K_{4}, k_{4} \\
\mathrm{Co}(\mathrm{III})-\mathrm{H}+\mathrm{Co}(\mathrm{III})-\mathrm{H} \rightarrow 2 \mathrm{Co}(\mathrm{II})+\mathrm{H}_{2} \quad K_{7}, k_{7} \\
\frac{d[\mathrm{Co}(\mathrm{III})-\mathrm{H}]}{d t}=k_{4}[\mathrm{Co}(\mathrm{I})][\mathrm{HA}]-k_{-4}[\mathrm{Co}(\mathrm{III})-\mathrm{H}][\mathrm{A}]-2 k_{7}[\mathrm{Co}(\mathrm{III})-\mathrm{H}]^{2} \\
\frac{d[\mathrm{Co}(\mathrm{II})]}{d t}=2 k_{7}[\mathrm{Co}(\mathrm{III})-\mathrm{H}]^{2}=2 \frac{d\left[\mathrm{H}_{2}\right]}{d t}
\end{gathered}
$$

Assuming a steady state for $\mathrm{Co}(\mathrm{III})-\mathrm{H}$

$$
2 k_{7}[\mathrm{Co}(\mathrm{III})-\mathrm{H}]^{2}+k_{-4}[\mathrm{Co}(\mathrm{III})-\mathrm{H}][\mathrm{HA}]-k_{4}[\mathrm{Co}(\mathrm{I})][\mathrm{HA}]=0
$$

solving we obtain,

$$
[\mathrm{Co}(\mathrm{III})-\mathrm{H}]=\frac{-k_{-4}[\mathrm{~A}] \pm \sqrt{\left(k_{-4}[\mathrm{~A}]\right)^{2}+8 k_{7} k_{4}[\mathrm{Co}(\mathrm{I})][\mathrm{HA}]}}{2 k_{7}}
$$

If the first term in the square root is larger then second, $\left(k_{-4}[\mathrm{~A}]\right)^{2}>>8 k_{7} k_{4}[\mathrm{Co}(\mathrm{I})][\mathrm{HA}]$, then,

$$
\begin{aligned}
& {[\mathrm{Co}(\mathrm{III})-\mathrm{H}]=\frac{-k_{-4}[\mathrm{~A}]+k_{-4}[\mathrm{~A}] \sqrt{1+\frac{8 k_{7} k_{4}[\mathrm{Co}(\mathrm{I})][\mathrm{HA}]}{\left(k_{-4}[\mathrm{~A}]\right)^{2}}}}{4 k_{7}}} \\
& \approx \frac{-k_{-4}[\mathrm{~A}]+k_{-4}[\mathrm{~A}]+\frac{1}{2} \frac{8 k_{-4} k_{7} k_{4}[\mathrm{Co}(\mathrm{I})][\mathrm{HA}]}{\left(k_{-4}[\mathrm{~A}]\right)^{2}}}{4 k_{7}} \\
& {[\mathrm{Co}(\mathrm{III})-\mathrm{H}] \approx \frac{1}{2} \frac{8 k_{7} k_{4}[\mathrm{Co}(\mathrm{I})][\mathrm{HA}]}{4 k_{7} k_{-4}[\mathrm{~A}]}} \\
& =\frac{k_{4}[\mathrm{Co}(\mathrm{I})][\mathrm{HA}]}{k_{-4}[\mathrm{~A}]} \\
& =K_{4} \frac{[\mathrm{Co}(\mathrm{I})][\mathrm{HA}]}{[\mathrm{A}]} \\
& \frac{d\left[\mathrm{H}_{2}\right]}{d t}=k_{7} K_{4}{ }^{2} \frac{[\mathrm{CoI}]^{2}[\mathrm{HA}]^{2}}{[\mathrm{~A}]^{2}}
\end{aligned}
$$

The rate is second order in $[\mathrm{Co}(\mathrm{I})]$ and $[\mathrm{HA}]$ and inverse in $[\mathrm{A}]^{2}$ with a rate constant as the product of the equilibrium constant for the rapid formation of the precursor hydride and the rate constant of the slow hydrogen formation reaction.

If second term is larger, $\left(k_{-4}[\mathrm{~A}]\right)^{2}<<8 k_{7} k_{4}[\mathrm{Co}(\mathrm{I})][\mathrm{HA}]$, then:

$$
[\mathrm{Co}(\mathrm{III})-\mathrm{H}]=\frac{-k_{-4}[\mathrm{~A}]+\sqrt{8 k_{7} k_{4}[\mathrm{Co}(\mathrm{I})][\mathrm{HA}]} \sqrt{1+\frac{\left(k_{-4}[\mathrm{~A}]\right)^{2}}{4 k_{7} k_{4}[\mathrm{Co}(\mathrm{I})][\mathrm{HA}]}}}{4 k_{7}}
$$




$$
\begin{gathered}
\approx \frac{\sqrt{8 k_{7} k_{4}[\mathrm{Co}(\mathrm{I})][\mathrm{HA}]}-k_{-4}[\mathrm{~A}]}{4 k_{7}} \\
=\sqrt{\frac{k_{4}[\mathrm{Co}(\mathrm{I})][\mathrm{HA}]}{2 k_{7}}} \\
\frac{d\left[\mathrm{H}_{2}\right]}{d t} \\
=k_{7}\left(\sqrt{\frac{k_{4}[\mathrm{Co}(\mathrm{I})][\mathrm{HA}]}{2 k_{7}}}\right)^{2} \\
=k_{4}[\mathrm{Co}(\mathrm{I})][\mathrm{HA}]
\end{gathered}
$$

The rate is first order in $[\mathrm{Co}(\mathrm{I})]$ and $[\mathrm{HA}]$ with a rate constant of the show hydride formation reaction. 


\section{Electrocatalysis of $4 \mathrm{a}$ in the presence of $\mathrm{HBF}_{4} \cdot \mathrm{Et}_{2} \mathrm{O}$.}

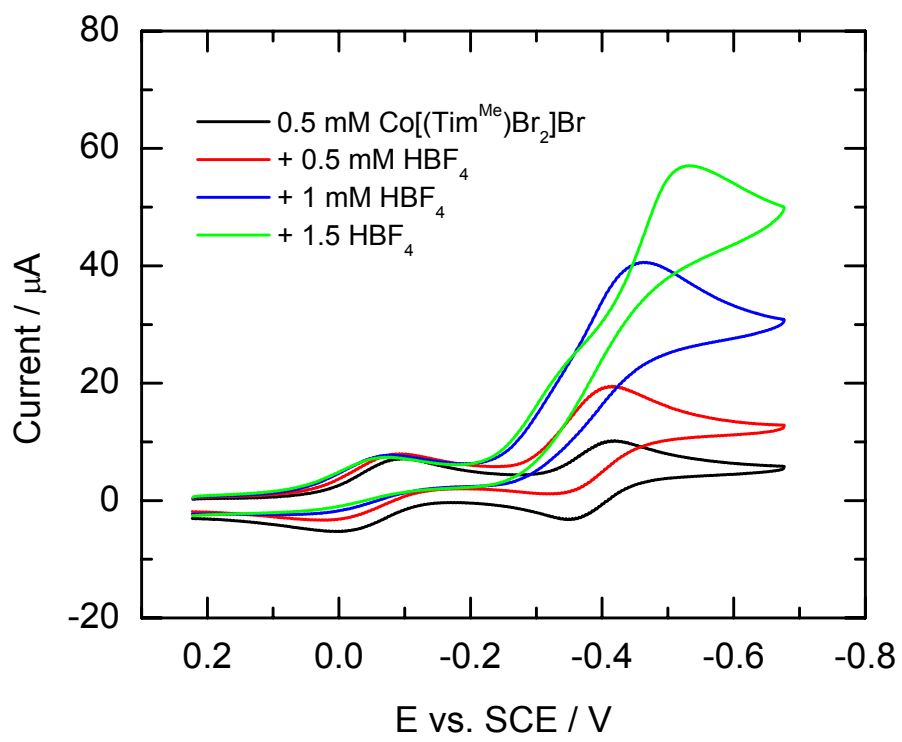

Figure S1. Cyclic voltammogram of $4 \mathbf{a}$ in the presence of $\mathrm{HBF}_{4} \cdot \mathrm{Et}_{2} \mathrm{O}$ recorded in an acetonitrile solution containing $0.1 \mathrm{M}\left[{ }^{\mathrm{n}} \mathrm{Bu}_{4}\right]\left[\mathrm{ClO}_{4}\right]$. Scan rate: $100 \mathrm{mV} / \mathrm{s}$. 
Electrocatalysis of $4 \mathrm{~b}$ in the presence of $\mathrm{TsOH} \cdot \mathrm{H}_{2} \mathrm{O}$ or $\mathrm{HBF}_{4} \cdot \mathrm{Et}_{2} \mathrm{O}$.
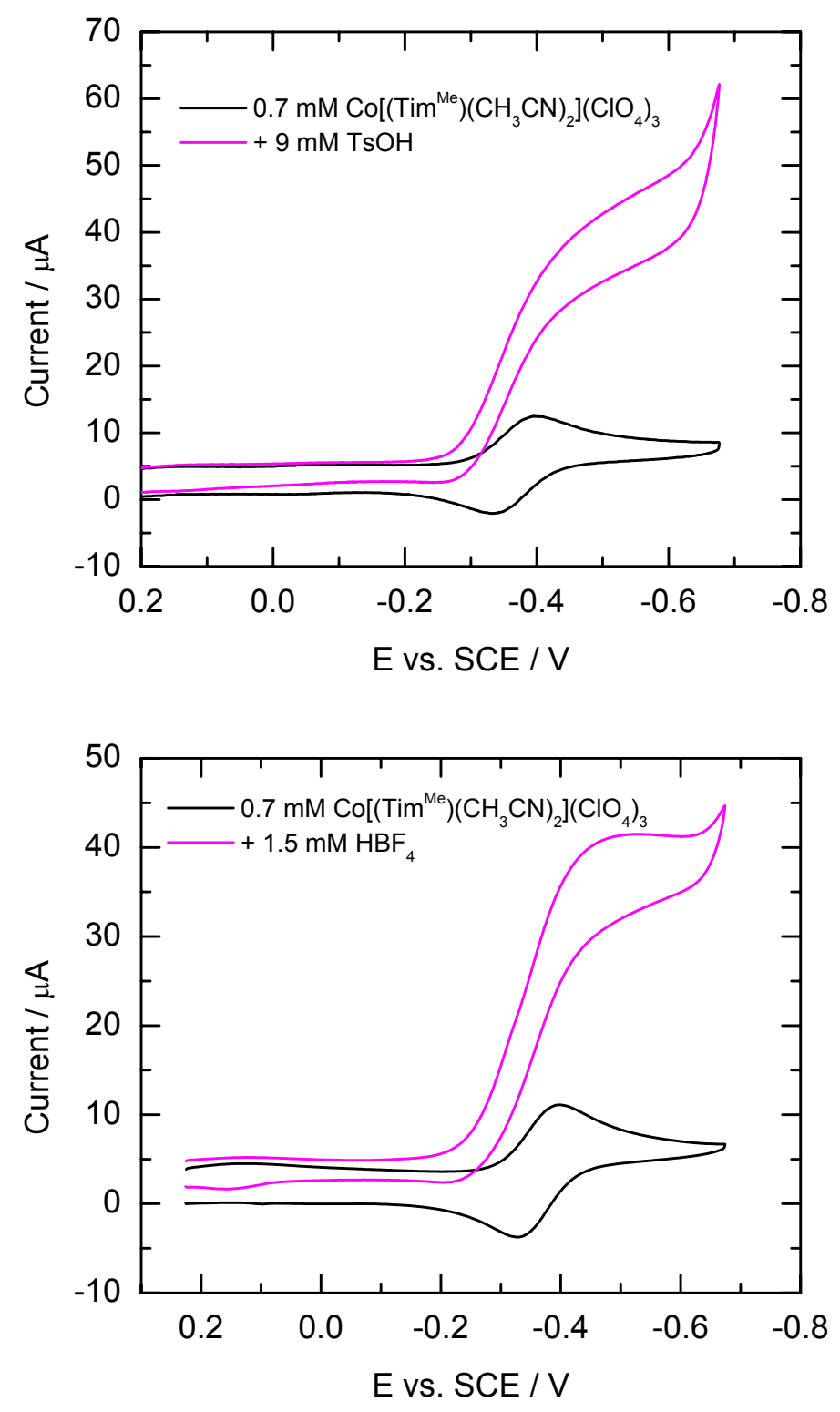

Figure S2. Cyclic voltammogram of $\mathbf{4} \mathbf{b}$ in the presence of $\mathrm{TsOH} \cdot \mathrm{H}_{2} \mathrm{O}$ (top) or $\mathrm{HBF}_{4} \cdot \mathrm{Et}_{2} \mathrm{O}$ (bottom) recorded in an acetonitrile solution containing $0.1 \mathrm{M}\left[{ }^{\mathrm{n}} \mathrm{Bu}_{4}\right]\left[\mathrm{ClO}_{4}\right]$. Scan rate: $100 \mathrm{mV} / \mathrm{s}$. 


\section{Electrocatalysis of $5 a-b$ in the presence of $\mathrm{HBF}_{4} \cdot \mathrm{Et}_{2} \mathrm{O}$.}
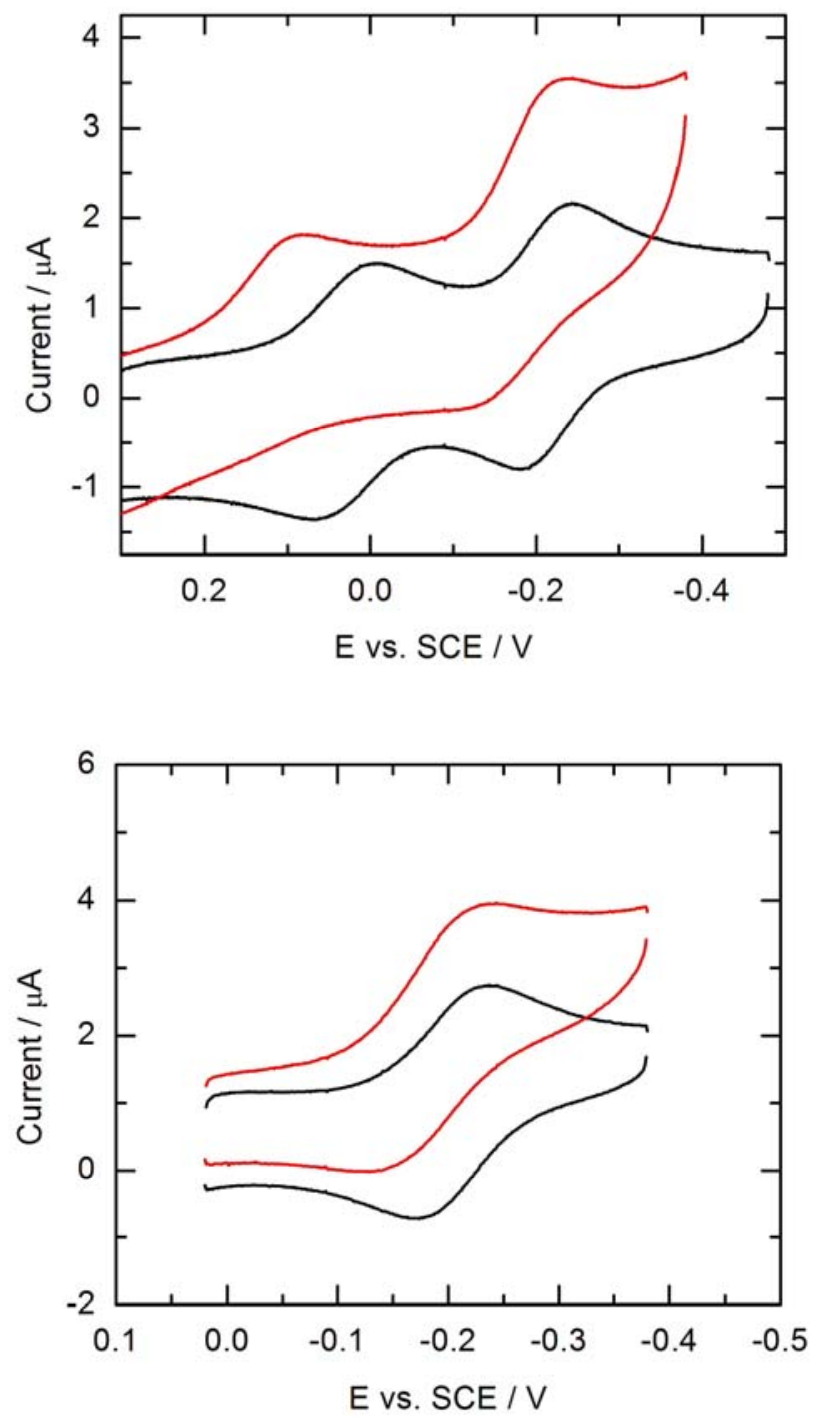

Figure S3. (Top): Cyclic voltammogram of $0.1 \mathrm{mM} 5 \mathbf{a}$ in $\mathrm{CH}_{3} \mathrm{CN}$ solution containing 0.1 $\mathrm{M}\left[{ }^{\mathrm{n}} \mathrm{Bu}_{4} \mathrm{~N}\right]\left[\mathrm{ClO}_{4}\right]$ in the absence (black) and presence (red) of $1.5 \mathrm{mM} \mathrm{HBF}_{4} \bullet \mathrm{Et}_{2} \mathrm{O}$. (Bottom): Cyclic voltammogram of $0.15 \mathrm{mM} 5 \mathbf{b}$ in $\mathrm{CH}_{3} \mathrm{CN}$ solution containing $0.1 \mathrm{M}$ $\left[{ }^{\mathrm{n}} \mathrm{Bu}_{4} \mathrm{~N}\right]\left[\mathrm{ClO}_{4}\right]$ in the absence (black) and presence (red) of $1.5 \mathrm{mM} \mathrm{HBF}_{4} \cdot \mathrm{Et}_{2} \mathrm{O}$. Scan rate: $100 \mathrm{mV} / \mathrm{s}^{-1}$; Glassy carbon electrode. The reaction for the shift in the $\mathrm{Co}^{\mathrm{III} / \mathrm{II}}$ potential upon addition of acid is not clear at this point. 

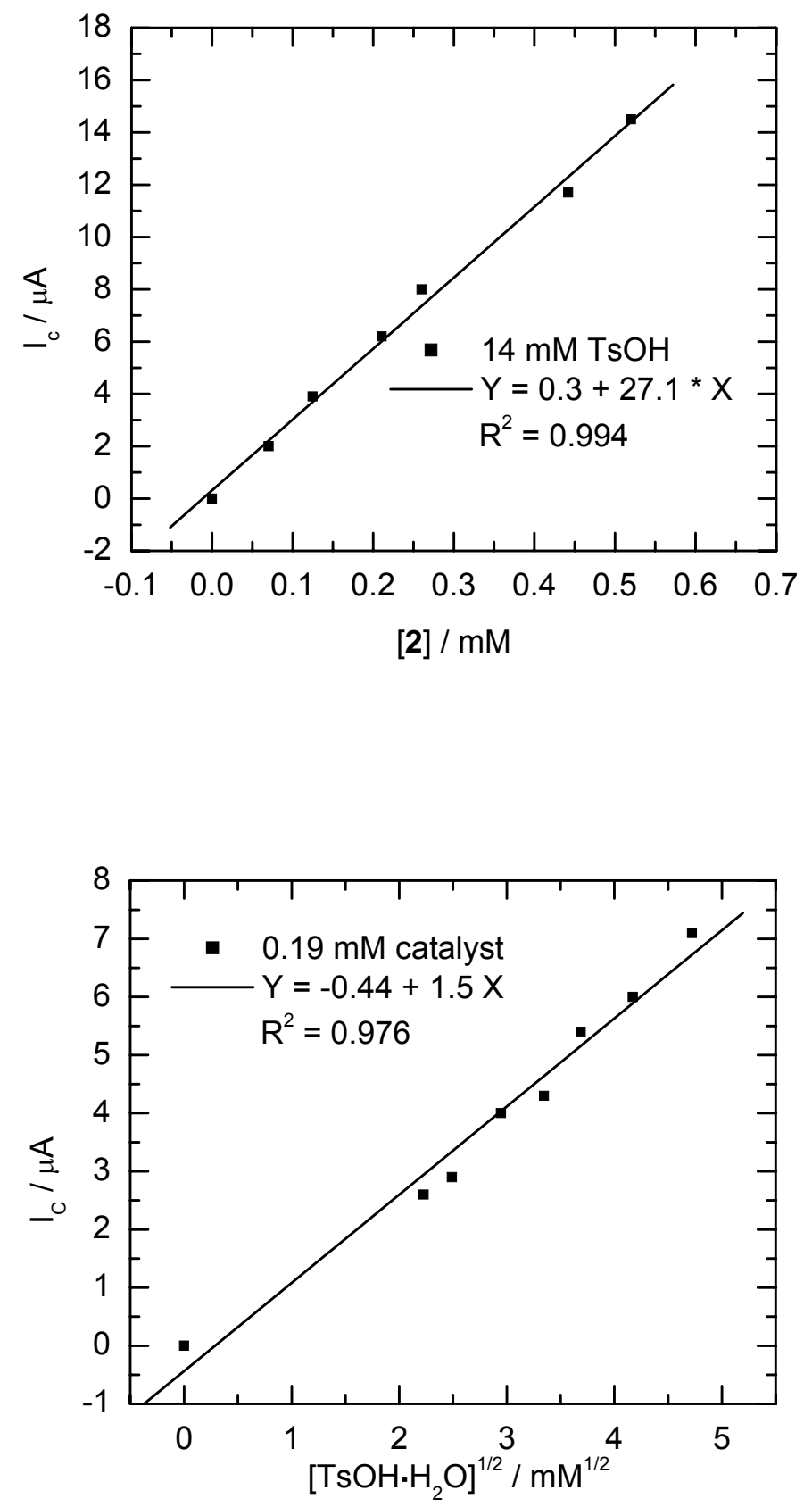

Figure S4. Dependence of the catalytic current $i_{c}$ on the concentrations of catalyst and acid for complex 2; $\mathrm{TsOH} \bullet \mathrm{H}_{2} \mathrm{O}$ was used as the acid, with a scan rate of $100 \mathrm{mV} / \mathrm{s}$. 

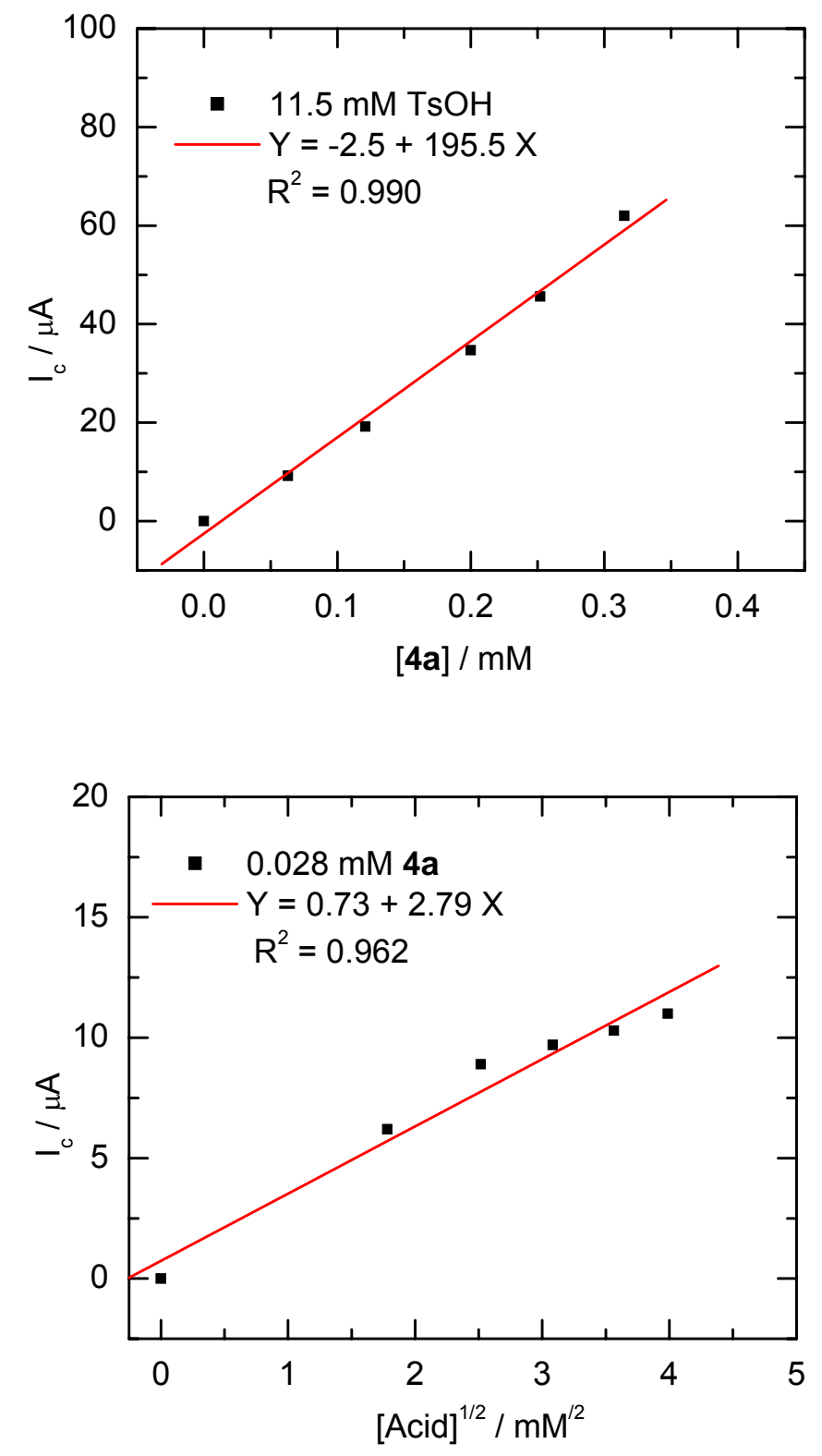

Figure S5. Dependence of the catalytic current $i_{c}$ on the concentrations of catalyst and acid for complex $\mathbf{4 a}$; $\mathrm{TsOH} \bullet \mathrm{H}_{2} \mathrm{O}$ was used as the acid. 

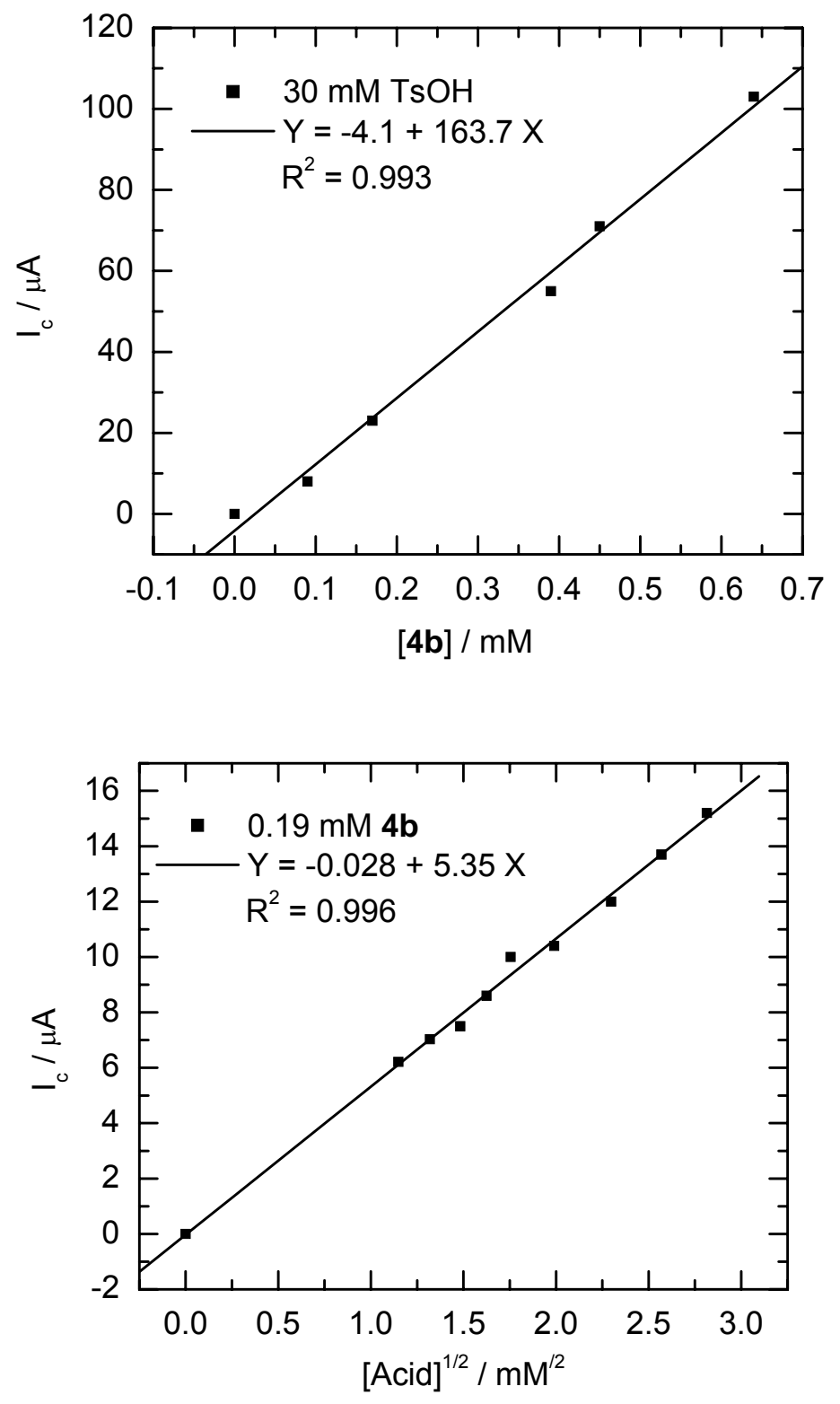

Figure S6. Dependence of the catalytic current $i_{c}$ on the concentrations of catalyst and acid for complex $\mathbf{4 b} ; \mathrm{TsOH} \bullet \mathrm{H}_{2} \mathrm{O}$ was used as the acid. 

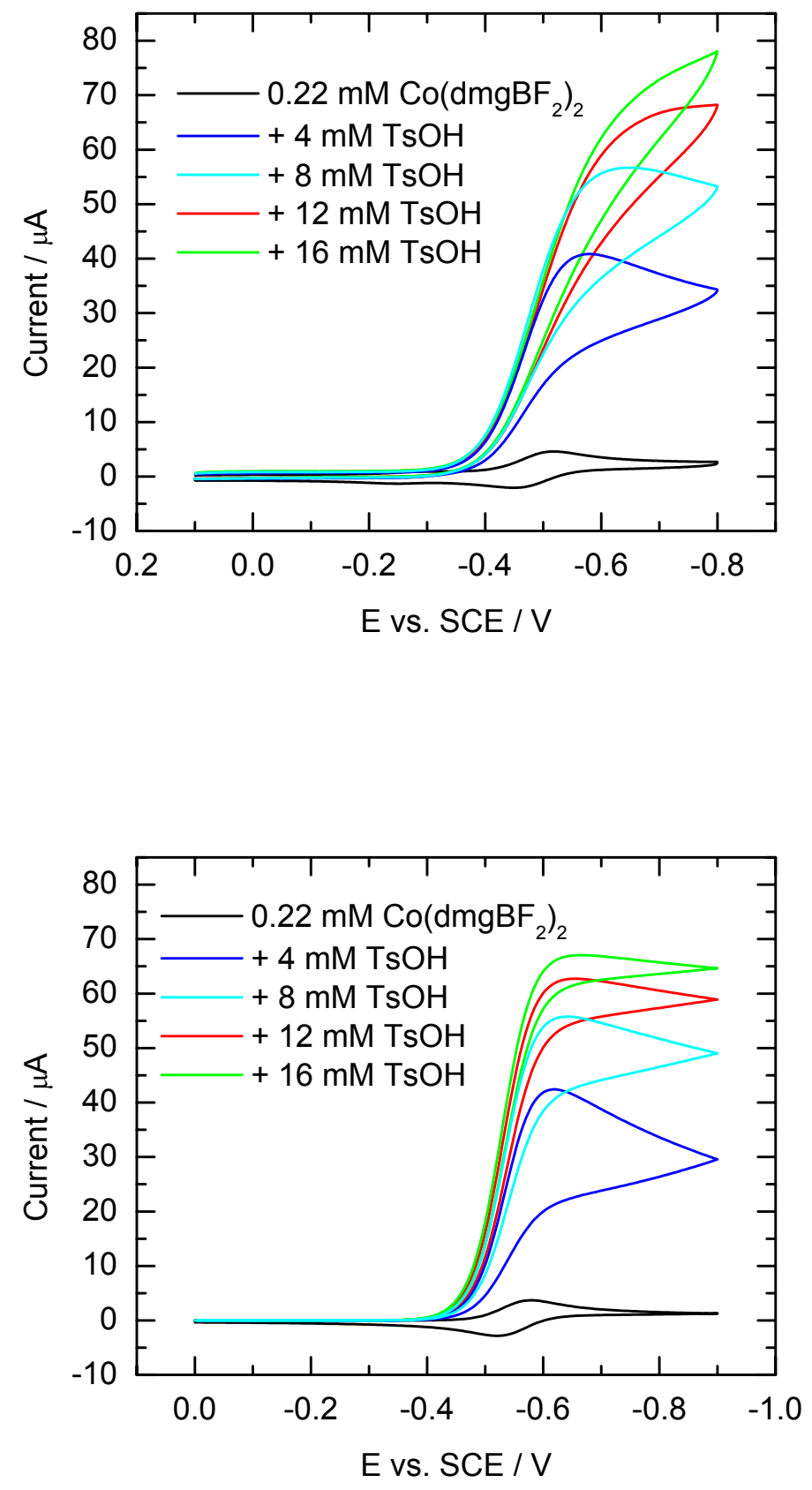
Figure S7. Experimental and simulated spectra of the catalytic waves for $\mathrm{H}_{2}$ evolution catalysis using $0.22 \mathrm{mM}$ of $\mathbf{1}$ as the catalyst; top: experimental data; bottom: simulation using a bimetallic mechanism.

\section{Specific Parameters for Simulation in Figure S7:}

\begin{tabular}{|c|c|c|}
\hline Estart (V) & Erev (V) & Eend (V) \\
\hline 0 & -0.9 & 0 \\
\hline
\end{tabular}

\section{Charge Transfer reactions:}

\begin{tabular}{|c|c|c|c|}
\hline Rxn & E (V) & $\alpha$ & $\mathrm{Ks}(\mathrm{cm} / \mathrm{s})$ \\
\hline $\mathrm{Co}(\mathrm{III})+\mathrm{e}^{-} \rightarrow \mathrm{Co}(\mathrm{II})$ & 0.2 & 0.5 & 10000 \\
\hline $\mathrm{Co}(\mathrm{II})+\mathrm{e}^{-} \rightarrow \mathrm{Co}(\mathrm{II})$ & -0.55 & 0.5 & 10000 \\
\hline $\mathrm{H}^{+}+\mathrm{e}^{-} \rightarrow \mathrm{s}$ & -0.23 & 0.5 & 0 \\
\hline
\end{tabular}

\section{Chemical reactions:}

\begin{tabular}{|c|c|c|c|}
\hline Rxn & Keq & $\mathrm{kf}$ & $\mathrm{kb}^{*}$ \\
\hline $\mathrm{Co}(\mathrm{I})+\mathrm{H}^{+} \rightarrow \mathrm{Co}-\mathrm{H}$ & $1 \mathrm{e} 5$ & $1.5 \mathrm{e} 5$ & 1.5 \\
\hline $\mathrm{s}+\mathrm{s} \rightarrow \mathrm{H}_{2}$ & 1 & $1 \mathrm{e} 10$ & $1 \mathrm{e} 10$ \\
\hline $\mathrm{Co}-\mathrm{H}+\mathrm{Co}-\mathrm{H} \rightarrow \mathrm{A}+\mathrm{H}_{2}$ & 1 & $1.5 \mathrm{e} 6$ & $1.5 \mathrm{e} 6$ \\
\hline $\mathrm{A} \rightarrow \mathrm{Co}(\mathrm{II})+\mathrm{Co}(\mathrm{II})$ & $6.55^{*}$ & $1 \mathrm{e} 10$ & $1.53 \mathrm{e} 9$ \\
\hline
\end{tabular}

* These constants are automatically calculated by the program

\section{Species:}

\begin{tabular}{|l|l|l|l|l|l|l|l|l|}
\hline & $\mathrm{D}\left(\mathrm{cm}^{2} / \mathrm{s}\right)$ & $\mathrm{C}(\mathrm{M})$ & & $\mathrm{D}\left(\mathrm{cm}^{2} / \mathrm{s}\right)$ & $\mathrm{C}(\mathrm{M})$ & & $\mathrm{D}\left(\mathrm{cm}^{2} / \mathrm{s}\right)$ & $\mathrm{C}(\mathrm{M})$ \\
\hline
\end{tabular}




\begin{tabular}{|c|c|c|c|c|c|c|c|c|}
\hline $\mathrm{Co}(\mathrm{III})$ & $8 \mathrm{e}-6$ & 0 & $\mathrm{Co}-\mathrm{H}$ & $8 \mathrm{e}-6$ & 0 & $\mathrm{~A}$ & $8 e-6$ & 0 \\
\hline$\overline{\mathrm{Co}(\mathrm{II})}$ & $8 \mathrm{e}-6$ & $2.2 \mathrm{e}-4$ & $\mathrm{H}_{2}$ & $1 \mathrm{e}-5$ & 0 & & & \\
\hline $\mathrm{Co}(\mathrm{I})$ & $8 \mathrm{e}-6$ & 0 & $\mathrm{~S}$ & $1 \mathrm{e}-5$ & 0 & & & \\
\hline
\end{tabular}

Acid concentrations, $\mathrm{H}^{+}$represents $\mathrm{TsOH}$

\begin{tabular}{|c|c|c|c|c|c|c|}
\hline & $\mathrm{D}\left(\mathrm{cm}^{2} / \mathrm{s}\right)$ & $\mathrm{C}(\mathrm{M})$ & $\mathrm{C}(\mathrm{M})$ & $\mathrm{C}(\mathrm{M})$ & $\mathrm{C}(\mathrm{M})$ & $\mathrm{C}(\mathrm{M})$ \\
\hline $\mathrm{H}^{+}$ & $1 \mathrm{e}-5$ & 0 & $4 \mathrm{e}-3$ & $8 \mathrm{e}-3$ & $12 \mathrm{e}-3$ & $16 \mathrm{e}-3$ \\
\hline
\end{tabular}



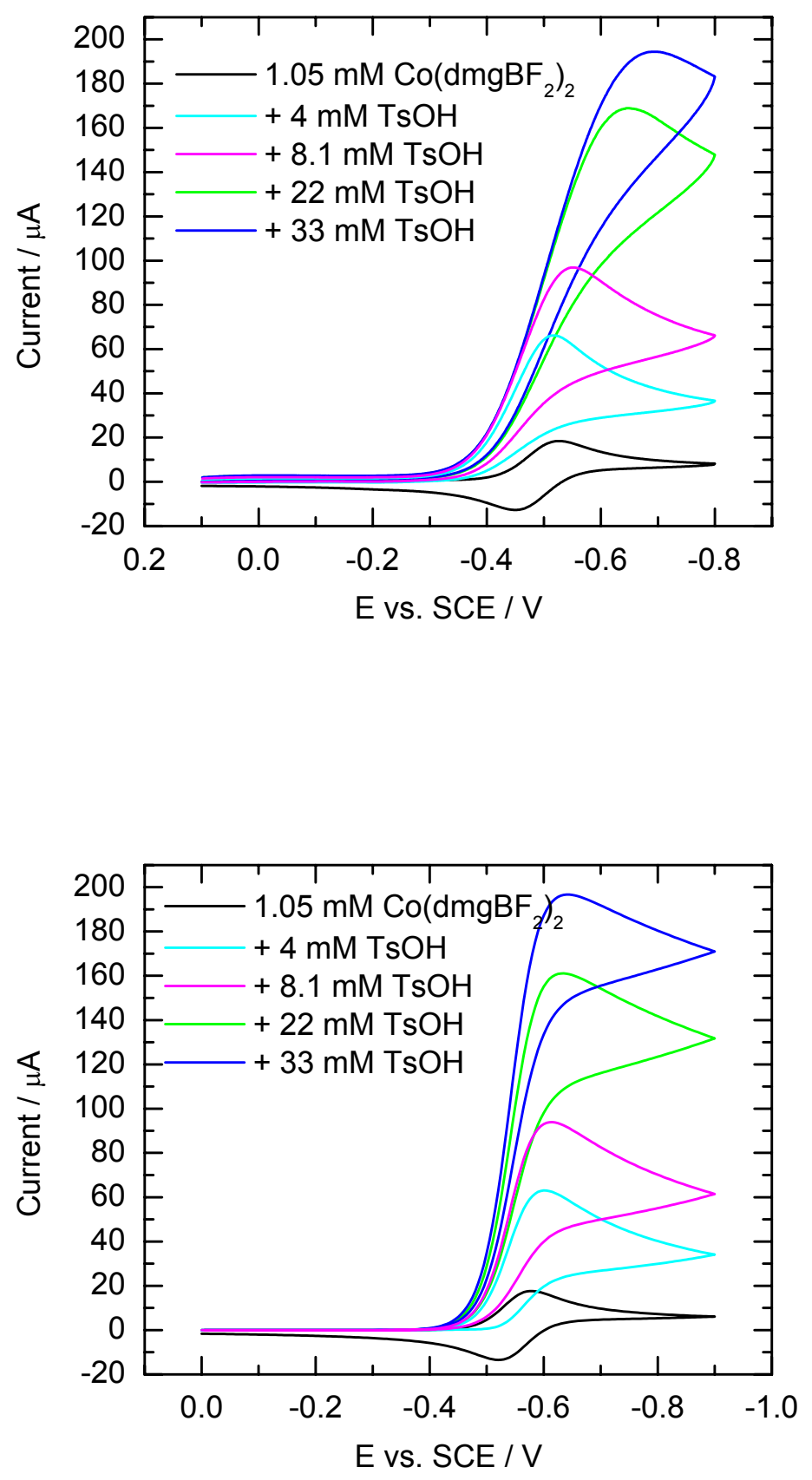
Figure S8. Experimental and simulated spectra of the catalytic waves for $\mathrm{H}_{2}$ evolution catalysis using $1.05 \mathrm{mM}$ of $\mathbf{1}$ as the catalyst; top: experimental data; bottom: simulation using a bimetallic mechanism.

Specific Parameters for Simulation in Figure S8:

\begin{tabular}{|c|c|c|}
\hline Estart (V) & Erev (V) & Eend (V) \\
\hline 0 & -0.9 & 0 \\
\hline
\end{tabular}

\section{Charge Transfer reactions:}

\begin{tabular}{|c|c|c|c|}
\hline Rxn & E (V) & $\alpha$ & $\mathrm{Ks}(\mathrm{cm} / \mathrm{s})$ \\
\hline $\mathrm{Co}(\mathrm{III})+\mathrm{e}^{-} \rightarrow \mathrm{Co}(\mathrm{II})$ & 0.2 & 0.5 & 10000 \\
\hline $\mathrm{Co}(\mathrm{II})+\mathrm{e}^{-} \rightarrow \mathrm{Co}(\mathrm{II})$ & -0.55 & 0.5 & 10000 \\
\hline $\mathrm{H}^{+}+\mathrm{e}^{-} \rightarrow \mathrm{s}$ & -0.23 & 0.5 & 0 \\
\hline
\end{tabular}

\section{Chemical reactions:}

\begin{tabular}{|c|c|c|c|}
\hline Rxn & Keq & $\mathrm{kf}$ & $\mathrm{kb}^{*}$ \\
\hline $\mathrm{Co}(\mathrm{I})+\mathrm{H}^{+} \rightarrow \mathrm{Co}-\mathrm{H}$ & $1 \mathrm{e} 5$ & 7000 & 0.07 \\
\hline $\mathrm{s}+\mathrm{s} \rightarrow \mathrm{H}_{2}$ & 1 & $1 \mathrm{e} 10$ & $1 \mathrm{e} 10$ \\
\hline $\mathrm{Co}-\mathrm{H}+\mathrm{Co}-\mathrm{H} \rightarrow \mathrm{A}+\mathrm{H}_{2}$ & 1 & $9 \mathrm{e} 5$ & $9 \mathrm{e} 5$ \\
\hline $\mathrm{A} \rightarrow \mathrm{Co}(\mathrm{II})+\mathrm{Co}(\mathrm{II})$ & $6.55^{*}$ & $1 \mathrm{e} 10$ & $1.53 \mathrm{e} 9$ \\
\hline
\end{tabular}

* These constants are automatically calculated by the program 


\section{Species:}

\begin{tabular}{|c|c|c|c|c|c|c|c|c|}
\hline & $\mathrm{D}\left(\mathrm{cm}^{2} / \mathrm{s}\right)$ & $\mathrm{C}(\mathrm{M})$ & & $\mathrm{D}\left(\mathrm{cm}^{2} / \mathrm{s}\right)$ & $\mathrm{C}(\mathrm{M})$ & & $\mathrm{D}\left(\mathrm{cm}^{2} / \mathrm{s}\right)$ & $\mathrm{C}(\mathrm{M})$ \\
\hline $\mathrm{Co}$ (III) & $8 e-6$ & 0 & $\mathrm{Co}-\mathrm{H}$ & $8 e-6$ & 0 & $\mathrm{~A}$ & $8 e-6$ & 0 \\
\hline $\mathrm{Co}(\mathrm{II})$ & $8 e-6$ & $1.05 \mathrm{e}-3$ & $\overline{\mathrm{H}_{2}}$ & $1 e-5$ & 0 & & & \\
\hline $\mathrm{Co}(\mathrm{I})$ & $8 e-6$ & 0 & $S$ & $1 e-5$ & 0 & & & \\
\hline
\end{tabular}

Acid concentrations, $\mathrm{H}^{+}$represents $\mathrm{TsOH}$

\begin{tabular}{|c|c|c|c|c|c|c|}
\hline & $\mathrm{D}\left(\mathrm{cm}^{2} / \mathrm{s}\right)$ & $\mathrm{C}(\mathrm{M})$ & $\mathrm{C}(\mathrm{M})$ & $\mathrm{C}(\mathrm{M})$ & $\mathrm{C}(\mathrm{M})$ & $\mathrm{C}(\mathrm{M})$ \\
\hline $\mathrm{H}^{+}$ & $1 \mathrm{e}-5$ & 0 & $4 \mathrm{e}-3$ & $8.1 \mathrm{e}-3$ & $22 \mathrm{e}-3$ & $33 \mathrm{e}-3$ \\
\hline
\end{tabular}



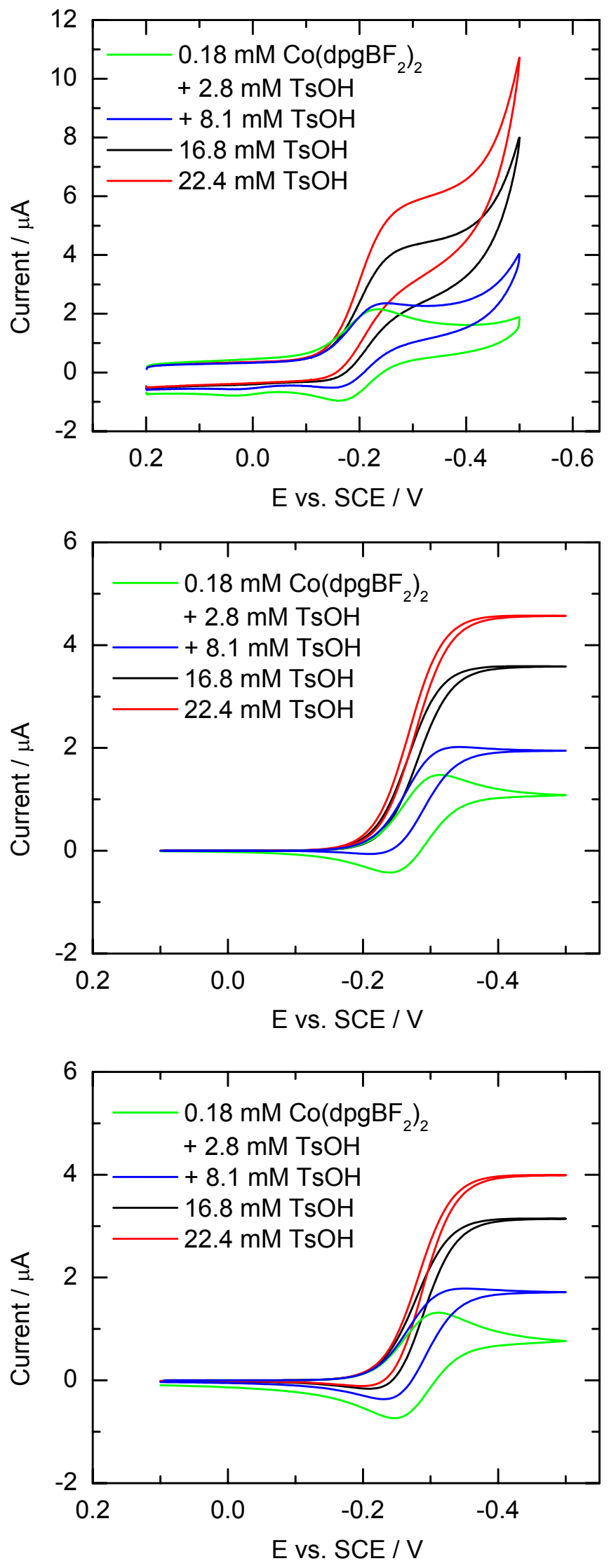
Figure S9. Experimental and simulated spectra of the catalytic waves for $\mathrm{H}_{2}$ evolution catalysis using $0.18 \mathrm{mM}$ of 2 as the catalyst; top: experimental data (the increase of current near $\mathrm{E}=-0.6 \mathrm{~V}$ is due to catalytic current at the $\mathrm{Co}(\mathrm{III})-\mathrm{H} / \mathrm{Co}(\mathrm{II})-\mathrm{H}$ couple, see main text); middle: simulation using a monometallic mechanism; bottom: simulation using a bimetallic mechanism. As shown in the figure, the simulations can not distinguish a monometallic mechanism from a bimetallic mechanism.

Specific Parameters for Simulation in Figure S9:

\begin{tabular}{|c|c|c|}
\hline Estart (V) & Erev (V) & Eend (V) \\
\hline 0.1 & -0.5 & 0.1 \\
\hline
\end{tabular}

\section{Charge Transfer reactions:}

\begin{tabular}{|c|c|c|c|}
\hline Rxn & E (V) & $\alpha$ & Ks (cm/s) \\
\hline $\mathrm{Co}(\mathrm{III})+\mathrm{e}^{-} \rightarrow \mathrm{Co}(\mathrm{II})$ & 0.3 & 0.5 & 10000 \\
\hline $\mathrm{Co}(\mathrm{II})+\mathrm{e}^{-} \rightarrow \mathrm{Co}(\mathrm{II})$ & -0.28 & 0.5 & 10000 \\
\hline $\mathrm{H}^{+}+\mathrm{e}^{-} \rightarrow \mathrm{s}$ & -0.23 & 0.5 & 0 \\
\hline
\end{tabular}

\section{Chemical reactions for monometallic mechanism:}

\begin{tabular}{|c|c|c|c|}
\hline $\mathrm{Rxn}$ & $\mathrm{Keq}$ & $\mathrm{kf}$ & $\mathrm{kb}^{*}$ \\
\hline $\mathrm{Co}(\mathrm{I})+\mathrm{H}^{+} \rightarrow \mathrm{Co}-\mathrm{H}$ & 50 & 5000 & 100 \\
\hline $\mathrm{s}+\mathrm{s} \rightarrow \mathrm{H}_{2}$ & 1 & $1 \mathrm{e} 10$ & $1 \mathrm{e} 10$ \\
\hline $\mathrm{Co}-\mathrm{H}+\mathrm{H}^{+} \rightarrow \mathrm{Co}(\mathrm{III})+\mathrm{H}_{2}$ & 1.54 & 800 & 518 \\
\hline $\mathrm{Co}(\mathrm{III})+\mathrm{Co}(\mathrm{I}) \rightarrow \mathrm{Co}(\mathrm{II})+\mathrm{Co}(\mathrm{II})$ & $6 \mathrm{e} 9 *$ & $1 \mathrm{e} 10$ & 1.53 \\
\hline
\end{tabular}


Chemical reactions for bimetallic mechanism:

\begin{tabular}{|c|c|c|c|}
\hline Rxn & Keq & $\mathrm{kf}$ & $\mathrm{kb}^{*}$ \\
\hline $\mathrm{Co}(\mathrm{I})+\mathrm{H}^{+} \rightarrow \mathrm{Co}-\mathrm{H}$ & 10 & $4 \mathrm{e} 4$ & $4 \mathrm{e} 3$ \\
\hline $\mathrm{s}+\mathrm{s} \rightarrow \mathrm{H}_{2}$ & 1 & $1 \mathrm{e} 10$ & $1 \mathrm{e} 10$ \\
\hline $\mathrm{Co}-\mathrm{H}+\mathrm{Co}-\mathrm{H} \rightarrow \mathrm{A}+\mathrm{H}_{2}$ & $4900^{*}$ & $1 \mathrm{e} 6$ & 204 \\
\hline $\mathrm{A} \rightarrow \mathrm{Co}(\mathrm{II})+\mathrm{Co}(\mathrm{II})$ & $1 \mathrm{e} 6$ & $1 \mathrm{e} 10$ & $1 \mathrm{e} 4$ \\
\hline
\end{tabular}

* These constants are automatically calculated by the program

\section{Species:}

\begin{tabular}{|c|c|c|c|c|c|c|c|c|}
\hline & $\mathrm{D}\left(\mathrm{cm}^{2} / \mathrm{s}\right)$ & $\mathrm{C}(\mathrm{M})$ & & $\mathrm{D}\left(\mathrm{cm}^{2} / \mathrm{s}\right)$ & $\mathrm{C}(\mathrm{M})$ & & $\mathrm{D}\left(\mathrm{cm}^{2} / \mathrm{s}\right)$ & $\mathrm{C}(\mathrm{M})$ \\
\hline $\mathrm{Co}(\mathrm{III})$ & $1.4 \mathrm{e}-6$ & 0 & $\mathrm{Co}-\mathrm{H}$ & $1.4 \mathrm{e}-6$ & 0 & $\mathrm{~A}$ & $1.4 \mathrm{e}-6$ & 0 \\
\hline $\mathrm{Co}(\mathrm{II})$ & $1.4 \mathrm{e}-6$ & $1.8 \mathrm{e}-4$ & $\mathrm{H}_{2}$ & $1 e-5$ & 0 & & & \\
\hline $\mathrm{Co}(\mathrm{I})$ & $1.4 \mathrm{e}-6$ & 0 & $\mathrm{~s}$ & $1 \mathrm{e}-5$ & 0 & & & \\
\hline
\end{tabular}

Acid concentrations, $\mathrm{H}^{+}$represents $\mathrm{TsOH}$

\begin{tabular}{|c|c|c|c|c|c|c|}
\hline & $\mathrm{D}\left(\mathrm{cm}^{2} / \mathrm{s}\right)$ & $\mathrm{C}(\mathrm{M})$ & $\mathrm{C}(\mathrm{M})$ & $\mathrm{C}(\mathrm{M})$ & $\mathrm{C}(\mathrm{M})$ & $\mathrm{C}(\mathrm{M})$ \\
\hline $\mathrm{H}^{+}$ & $1 \mathrm{e}-5$ & 0 & $2.8 \mathrm{e}-3$ & $8.4 \mathrm{e}-3$ & $16.8 \mathrm{e}-3$ & $22.4 \mathrm{e}-3$ \\
\hline
\end{tabular}



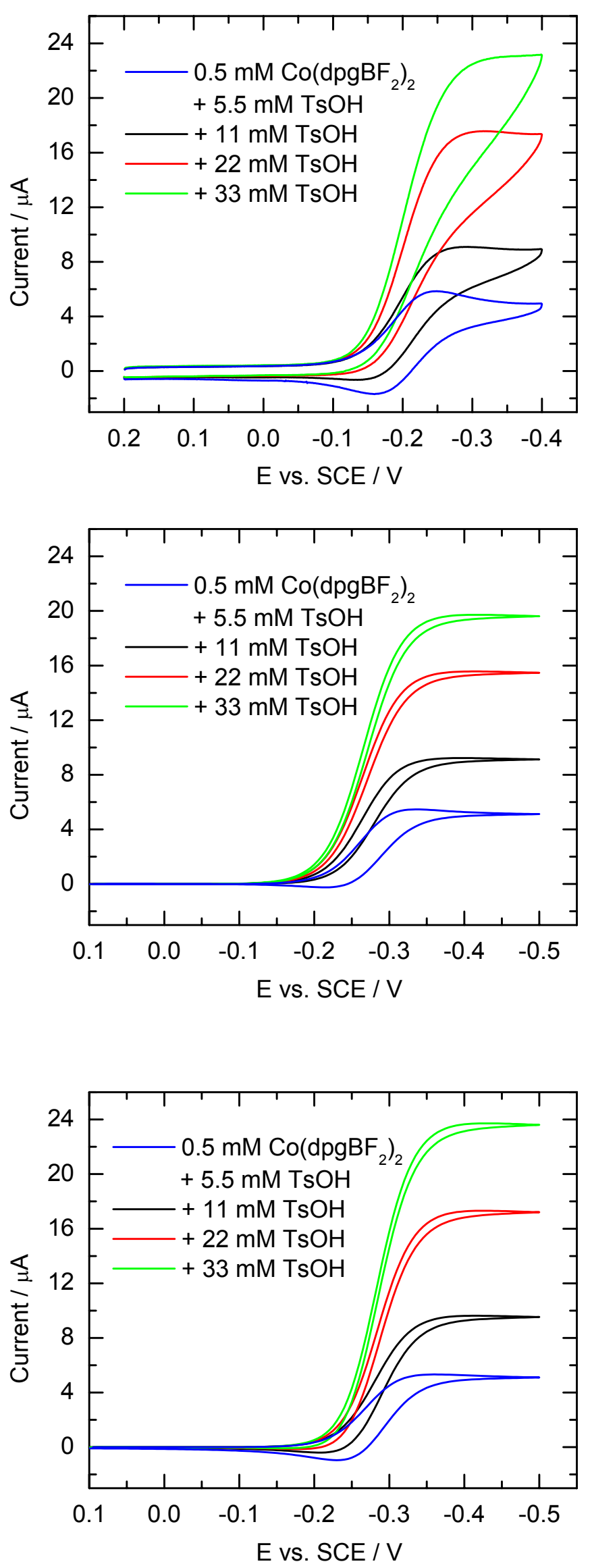
Figure S10. Experimental and simulated spectra of the catalytic waves for $\mathrm{H}_{2}$ evolution catalysis using $0.5 \mathrm{mM}$ of 2 as the catalyst; top: experimental data; middle: simulation using a monometallic mechanism; bottom: simulation using a bimetallic mechanism. As shown in the figure, the simulations can not distinguish a monometallic mechanism from a bimetallic mechanism.

\section{Specific Parameters for Simulation in Figure S10:}

\begin{tabular}{|c|c|c|}
\hline Estart (V) & Erev (V) & Eend (V) \\
\hline 0.1 & -0.5 & 0.1 \\
\hline
\end{tabular}

\section{Charge Transfer reactions:}

\begin{tabular}{|c|c|c|c|}
\hline Rxn & E (V) & $\alpha$ & $\mathrm{Ks}(\mathrm{cm} / \mathrm{s})$ \\
\hline $\mathrm{Co}(\mathrm{III})+\mathrm{e}^{-} \rightarrow \mathrm{Co}(\mathrm{II})$ & 0.3 & 0.5 & 10000 \\
\hline $\mathrm{Co}(\mathrm{II})+\mathrm{e}^{-} \rightarrow \mathrm{Co}(\mathrm{II})$ & -0.28 & 0.5 & 10000 \\
\hline $\mathrm{H}^{+}+\mathrm{e}^{-} \rightarrow \mathrm{s}$ & -0.23 & 0.5 & 0 \\
\hline
\end{tabular}

Chemical reactions for monometallic mechanism:

\begin{tabular}{|c|c|c|c|}
\hline Rxn & Keq & kf & $\mathrm{kb}^{*}$ \\
\hline $\mathrm{Co}(\mathrm{I})+\mathrm{H}^{+} \rightarrow \mathrm{Co}-\mathrm{H}$ & 50 & 5000 & 100 \\
\hline $\mathrm{s}+\mathrm{s} \rightarrow \mathrm{H}_{2}$ & 1 & $1 \mathrm{e} 10$ & $1 \mathrm{e} 10$ \\
\hline $\mathrm{Co}-\mathrm{H}+\mathrm{H}^{+} \rightarrow \mathrm{Co}(\mathrm{III})+\mathrm{H}_{2}$ & 1.54 & 800 & 518 \\
\hline $\mathrm{Co}(\mathrm{III})+\mathrm{Co}(\mathrm{I}) \rightarrow \mathrm{Co}(\mathrm{II})+\mathrm{Co}(\mathrm{II})$ & $6 \mathrm{e} 9^{*}$ & $1 \mathrm{e} 10$ & 1.53 \\
\hline
\end{tabular}

Chemical reactions for bimetallic mechanism: 


\begin{tabular}{|c|c|c|c|}
\hline Rxn & Keq & kf & $\mathrm{kb}^{*}$ \\
\hline $\mathrm{Co}(\mathrm{I})+\mathrm{H}^{+} \rightarrow \mathrm{Co}-\mathrm{H}$ & 10 & $4 \mathrm{e} 4$ & $4 \mathrm{e} 3$ \\
\hline $\mathrm{s}+\mathrm{s} \rightarrow \mathrm{H}_{2}$ & 1 & $1 \mathrm{e} 10$ & $1 \mathrm{e} 10$ \\
\hline $\mathrm{Co}-\mathrm{H}+\mathrm{Co}-\mathrm{H} \rightarrow \mathrm{A}+\mathrm{H}_{2}$ & $4900^{*}$ & $1 \mathrm{e} 6$ & 204 \\
\hline $\mathrm{A} \rightarrow \mathrm{Co}(\mathrm{II})+\mathrm{Co}(\mathrm{II})$ & $1 \mathrm{e} 6$ & $1 \mathrm{e} 10$ & $1 \mathrm{e} 4$ \\
\hline
\end{tabular}

* These constants are automatically calculated by the program

Species:

\begin{tabular}{|c|c|c|c|c|c|c|c|c|}
\hline & $\mathrm{D}\left(\mathrm{cm}^{2} / \mathrm{s}\right)$ & $\mathrm{C}(\mathrm{M})$ & & $\mathrm{D}\left(\mathrm{cm}^{2} / \mathrm{s}\right)$ & $\mathrm{C}(\mathrm{M})$ & & $\mathrm{D}\left(\mathrm{cm}^{2} / \mathrm{s}\right)$ & $\mathrm{C}(\mathrm{M})$ \\
\hline $\mathrm{Co}(\mathrm{III})$ & $1.4 \mathrm{e}-6$ & 0 & $\mathrm{Co}-\mathrm{H}$ & $1.4 \mathrm{e}-6$ & 0 & A & $1.4 \mathrm{e}-6$ & 0 \\
\hline $\mathrm{Co}(\mathrm{II})$ & $1.4 \mathrm{e}-6$ & $5 e-4$ & $\mathrm{H}_{2}$ & $1 e-5$ & 0 & & & \\
\hline $\mathrm{Co}(\mathrm{I})$ & $1.4 \mathrm{e}-6$ & 0 & $\mathrm{~s}$ & $1 \mathrm{e}-5$ & 0 & & & \\
\hline
\end{tabular}

Acid concentrations, $\mathrm{H}^{+}$represents $\mathrm{TsOH}$

\begin{tabular}{|c|c|c|c|c|c|c|}
\hline & $\mathrm{D}\left(\mathrm{cm}^{2} / \mathrm{s}\right)$ & $\mathrm{C}(\mathrm{M})$ & $\mathrm{C}(\mathrm{M})$ & $\mathrm{C}(\mathrm{M})$ & $\mathrm{C}(\mathrm{M})$ & $\mathrm{C}(\mathrm{M})$ \\
\hline $\mathrm{H}^{+}$ & $1 \mathrm{e}-5$ & 0 & $5.5 \mathrm{e}-3$ & $11 \mathrm{e}-3$ & $22 \mathrm{e}-3$ & $33 \mathrm{e}-3$ \\
\hline
\end{tabular}



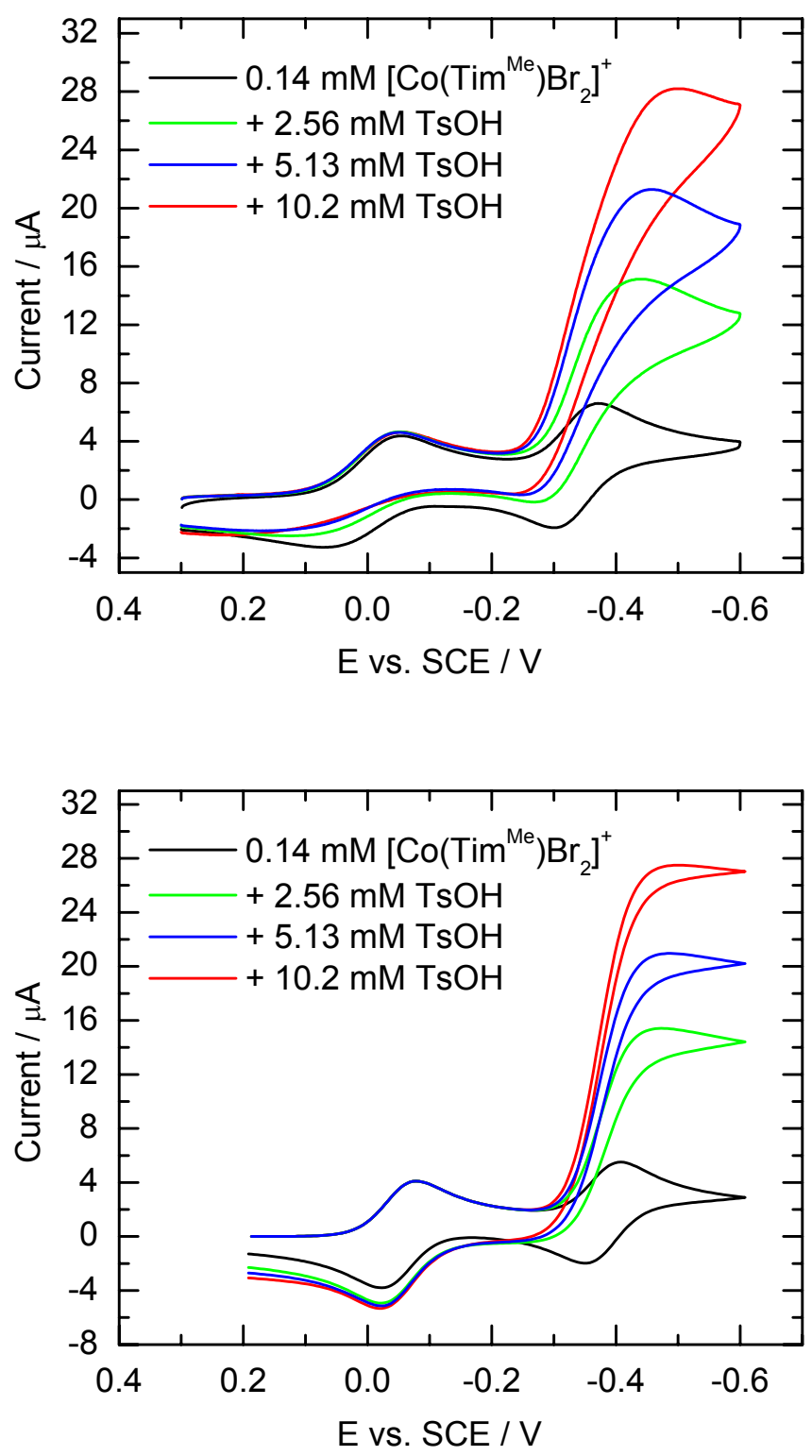

Figure S11. Experimental and simulated spectra of the catalytic waves for $\mathrm{H}_{2}$ evolution catalysis using $0.14 \mathrm{mM}$ of $\mathbf{4 a}$ as the catalyst; top: experimental data; bottom: simulation using a bimetallic mechanism. 
Specific Parameters for Simulation in Figure S11:

\begin{tabular}{|c|c|c|}
\hline Estart (V) & Erev (V) & Eend (V) \\
\hline 0.2 & -0.6 & 0.2 \\
\hline
\end{tabular}

\section{Charge Transfer reactions:}

\begin{tabular}{|c|c|c|c|}
\hline Rxn & $\mathrm{E}(\mathrm{V})$ & $\alpha$ & $\mathrm{Ks}(\mathrm{cm} / \mathrm{s})$ \\
\hline $\mathrm{Co}(\mathrm{III})+\mathrm{e}^{-} \rightarrow \mathrm{Co}(\mathrm{II})$ & -0.05 & 0.5 & 10000 \\
\hline $\mathrm{Co}(\mathrm{II})+\mathrm{e}^{-} \rightarrow \mathrm{Co}(\mathrm{II})$ & -0.38 & 0.5 & 10000 \\
\hline $\mathrm{H}^{+}+\mathrm{e}^{-} \rightarrow \mathrm{s}$ & -0.23 & 0.5 & 0 \\
\hline
\end{tabular}

\section{Chemical reactions:}

\begin{tabular}{|c|c|c|c|}
\hline Rxn & Keq & kf & $\mathrm{kb}^{*}$ \\
\hline $\mathrm{Co}(\mathrm{I})+\mathrm{H}^{+} \rightarrow \mathrm{Co}-\mathrm{H}$ & $1 \mathrm{e} 3$ & $1 \mathrm{e} 4$ & 10 \\
\hline $\mathrm{s}+\mathrm{s} \rightarrow \mathrm{H}_{2}$ & 1 & $1 \mathrm{e} 10$ & $1 \mathrm{e} 10$ \\
\hline $\mathrm{Co}-\mathrm{H}+\mathrm{Co}-\mathrm{H} \rightarrow \mathrm{A}+\mathrm{H}_{2}$ & 0.001 & $1 \mathrm{e} 6$ & $1 \mathrm{e} 9$ \\
\hline $\mathrm{A} \rightarrow \mathrm{Co}(\mathrm{II})+\mathrm{Co}(\mathrm{II})$ & $117.5^{*}$ & $1 \mathrm{e} 10$ & $8.5 \mathrm{e} 7$ \\
\hline
\end{tabular}

* These constants are automatically calculated by the program

\section{Species:}

\begin{tabular}{|c|c|c|c|c|c|c|c|c|}
\hline & $\mathrm{D}\left(\mathrm{cm}^{2} / \mathrm{s}\right)$ & $\mathrm{C}(\mathrm{M})$ & & $\mathrm{D}\left(\mathrm{cm}^{2} / \mathrm{s}\right)$ & C (M) & & $\mathrm{D}\left(\mathrm{cm}^{2} / \mathrm{s}\right)$ & C (M) \\
\hline $\mathrm{Co}(\mathrm{III})$ & $2.5 e-5$ & 0 & $\mathrm{Co}-\mathrm{H}$ & $2.5 e-5$ & 0 & A & $2.5 e-5$ & 0 \\
\hline $\mathrm{Co}(\mathrm{II})$ & $2.5 e-5$ & $1.38 \mathrm{e}-4$ & $\mathrm{H}_{2}$ & $1 e-5$ & 0 & & & \\
\hline $\mathrm{Co}(\mathrm{I})$ & $2.5 e-5$ & 0 & $\mathrm{~s}$ & $1 \mathrm{e}-5$ & 0 & & & \\
\hline
\end{tabular}


Acid concentrations, $\mathrm{H}^{+}$represents $\mathrm{TsOH}$

\begin{tabular}{|l|c|c|l|l|l|}
\hline & $\mathrm{D}\left(\mathrm{cm}^{2} / \mathrm{s}\right)$ & $\mathrm{C}(\mathrm{M})$ & $\mathrm{C}(\mathrm{M})$ & $\mathrm{C}(\mathrm{M})$ & $\mathrm{C}(\mathrm{M})$ \\
\hline $\mathrm{H}^{+}$ & $1 \mathrm{e}-5$ & 0 & $2.56 \mathrm{e}-3$ & $5.13 \mathrm{e}-3$ & $10.2 \mathrm{e}-3$ \\
\hline
\end{tabular}



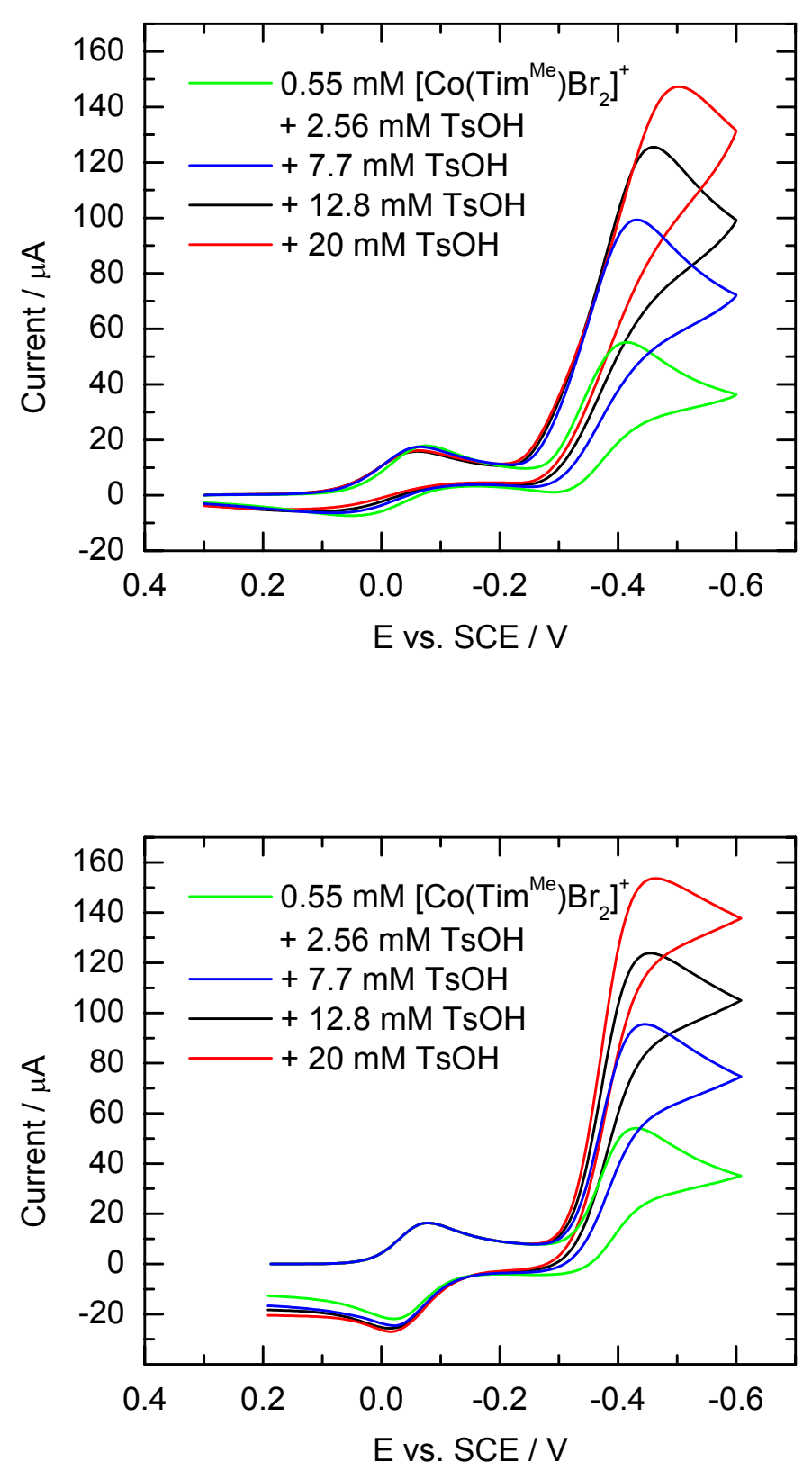

Figure S12. Experimental and simulated spectra of the catalytic waves for $\mathrm{H}_{2}$ evolution catalysis using $0.55 \mathrm{mM}$ of $\mathbf{4 a}$ as the catalyst; top: experimental data; bottom: simulation using a bimetallic mechanism. 
Specific Parameters for Simulation in Figure S12:

\begin{tabular}{|c|c|c|}
\hline Estart (V) & Erev (V) & Eend (V) \\
\hline 0.2 & -0.6 & 0.2 \\
\hline
\end{tabular}

\section{Charge Transfer reactions:}

\begin{tabular}{|c|c|c|c|}
\hline Rxn & E (V) & $\alpha$ & $\mathrm{Ks}(\mathrm{cm} / \mathrm{s})$ \\
\hline $\mathrm{Co}(\mathrm{III})+\mathrm{e}^{-} \rightarrow \mathrm{Co}(\mathrm{II})$ & -0.05 & 0.5 & 10000 \\
\hline $\mathrm{Co}(\mathrm{II})+\mathrm{e}^{-} \rightarrow \mathrm{Co}(\mathrm{II})$ & -0.38 & 0.5 & 10000 \\
\hline $\mathrm{H}^{+}+\mathrm{e}^{-} \rightarrow \mathrm{s}$ & -0.23 & 0.5 & 0 \\
\hline
\end{tabular}

\section{Chemical reactions:}

\begin{tabular}{|c|c|c|c|}
\hline $\mathrm{Rxn}$ & Keq & $\mathrm{kf}$ & $\mathrm{kb} *$ \\
\hline $\mathrm{Co}(\mathrm{I})+\mathrm{H}^{+} \rightarrow \mathrm{Co}-\mathrm{H}$ & $1 \mathrm{e} 3$ & $1 \mathrm{e} 4$ & 10 \\
\hline $\mathrm{s}+\mathrm{s} \rightarrow \mathrm{H}_{2}$ & 1 & $1 \mathrm{e} 10$ & $1 \mathrm{e} 10$ \\
\hline $\mathrm{Co}-\mathrm{H}+\mathrm{Co}-\mathrm{H} \rightarrow \mathrm{A}+\mathrm{H}_{2}$ & 0.001 & $1 \mathrm{e} 6$ & $1 \mathrm{e} 9$ \\
\hline $\mathrm{A} \rightarrow \mathrm{Co}(\mathrm{II})+\mathrm{Co}(\mathrm{II})$ & $117.5^{*}$ & $1 \mathrm{e} 10$ & $8.5 \mathrm{e} 7$ \\
\hline
\end{tabular}

* These constants are automatically calculated by the program

\section{Species:}

\begin{tabular}{|c|c|c|c|c|c|c|c|c|}
\hline & $\mathrm{D}\left(\mathrm{cm}^{2} / \mathrm{s}\right)$ & C (M) & & $\mathrm{D}\left(\mathrm{cm}^{2} / \mathrm{s}\right)$ & $\mathrm{C}(\mathrm{M})$ & & $\mathrm{D}\left(\mathrm{cm}^{2} / \mathrm{s}\right)$ & $\mathrm{C}(\mathrm{M})$ \\
\hline $\mathrm{Co}(\mathrm{III})$ & $2.5 e-5$ & 0 & Co-H & $2.5 e-5$ & 0 & A & $2.5 e-5$ & 0 \\
\hline $\mathrm{Co}(\mathrm{II})$ & $2.5 e-5$ & $5.5 \mathrm{e}-4$ & $\mathrm{H}_{2}$ & $1 \mathrm{e}-5$ & 0 & & & \\
\hline
\end{tabular}




\begin{tabular}{|l|l|l|l|l|l|}
\hline $\operatorname{Co}(\mathrm{I})$ & $2.5 \mathrm{e}-5$ & 0 & $\mathrm{~s}$ & $1 \mathrm{e}-5$ & 0 \\
\hline
\end{tabular}

Acid concentrations, $\mathrm{H}^{+}$represents $\mathrm{TsOH}$

\begin{tabular}{|l|c|c|c|c|c|}
\hline & $\mathrm{D}\left(\mathrm{cm}^{2} / \mathrm{s}\right)$ & $\mathrm{C}(\mathrm{M})$ & $\mathrm{C}(\mathrm{M})$ & $\mathrm{C}(\mathrm{M})$ & $\mathrm{C}(\mathrm{M})$ \\
\hline $\mathrm{H}^{+}$ & $1 \mathrm{e}-5$ & $2.56 \mathrm{e}-3$ & $7.7 \mathrm{e}-3$ & $12.8 \mathrm{e}-3$ & $20 \mathrm{e}-3$ \\
\hline
\end{tabular}



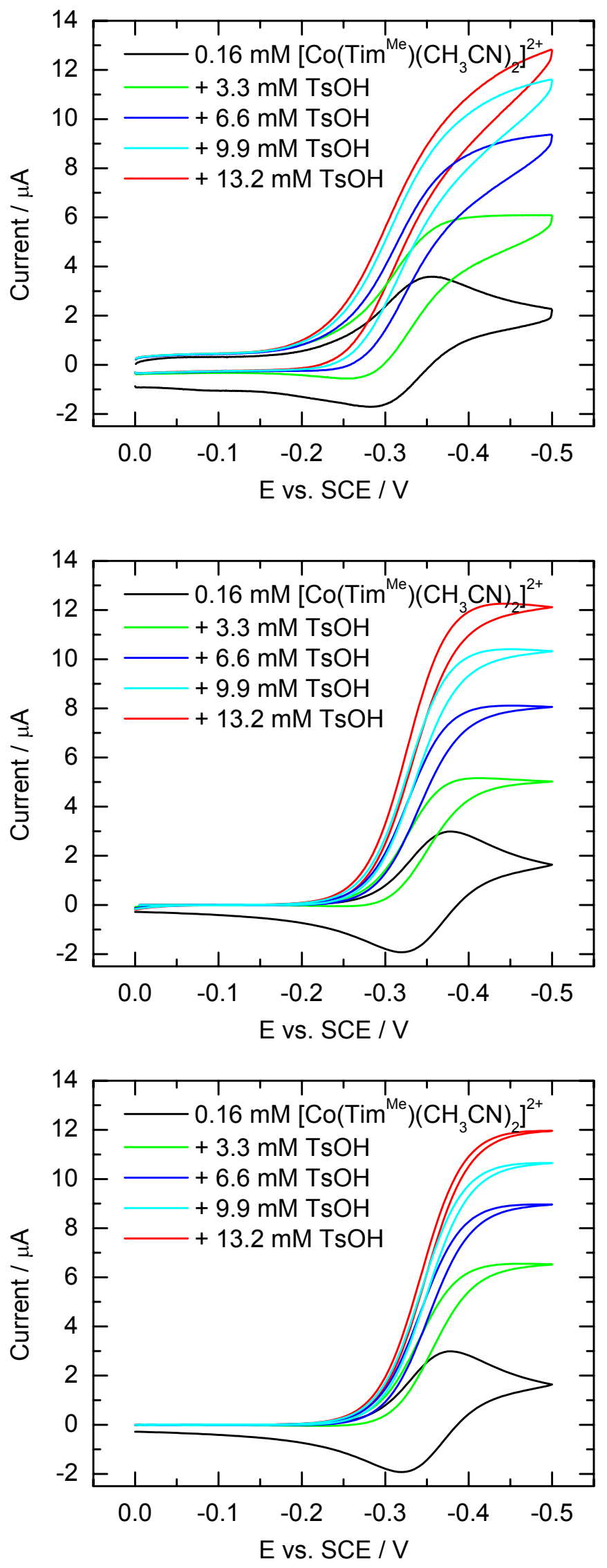
Figure S13. Experimental and simulated spectra of the catalytic waves for $\mathrm{H}_{2}$ evolution catalysis using $0.16 \mathrm{mM}$ of $\mathbf{4 b}$ as the catalyst; top: experimental data; middle: simulation using a monometallic mechanism; bottom: simulation using a bimetallic mechanism. As shown in the figure, the simulations can not distinguish a monometallic mechanism from a bimetallic mechanism.

Specific Parameters for Simulation in Figure S13:

\begin{tabular}{|c|c|c|}
\hline Estart (V) & Erev (V) & Eend (V) \\
\hline 0 & -0.5 & 0 \\
\hline
\end{tabular}

Charge Transfer reactions:

\begin{tabular}{|c|c|c|c|}
\hline Rxn & $\mathrm{E}(\mathrm{V})$ & $\alpha$ & $\mathrm{Ks}(\mathrm{cm} / \mathrm{s})$ \\
\hline $\mathrm{Co}(\mathrm{III})+\mathrm{e}^{-} \rightarrow \mathrm{Co}(\mathrm{II})$ & 0.2 & 0.5 & 10000 \\
\hline $\mathrm{Co}(\mathrm{II})+\mathrm{e}^{-} \rightarrow \mathrm{Co}(\mathrm{II})$ & -0.35 & 0.5 & 10000 \\
\hline $\mathrm{H}^{+}+\mathrm{e}^{-} \rightarrow \mathrm{s}$ & -0.23 & 0.5 & 0 \\
\hline
\end{tabular}

Chemical reactions for monometallic mechanism:

\begin{tabular}{|c|c|c|c|}
\hline Rxn & Keq & $\mathrm{kf}$ & $\mathrm{kb}^{*}$ \\
\hline $\mathrm{Co}(\mathrm{I})+\mathrm{H}^{+} \rightarrow \mathrm{Co}-\mathrm{H}$ & 300 & 8000 & 26.6 \\
\hline $\mathrm{s}+\mathrm{s} \rightarrow \mathrm{H}_{2}$ & 1 & $1 \mathrm{e} 10$ & $1 \mathrm{e} 10$ \\
\hline $\mathrm{Co}-\mathrm{H}+\mathrm{H}^{+} \rightarrow \mathrm{Co}(\mathrm{III})+\mathrm{H}_{2}$ & $1.9 \mathrm{e}-8$ & 650 & $4 \mathrm{e} 10$ \\
\hline $\mathrm{Co}(\mathrm{III})+\mathrm{Co}(\mathrm{I}) \rightarrow \mathrm{Co}(\mathrm{II})+\mathrm{Co}(\mathrm{II})$ & $1.9 \mathrm{e} 9 *$ & $1 \mathrm{e} 10$ & 5 \\
\hline
\end{tabular}


Chemical reactions for bimetallic mechanism:

\begin{tabular}{|c|c|c|c|}
\hline $\mathrm{Rxn}$ & $\mathrm{Keq}$ & $\mathrm{kf}$ & $\mathrm{kb}^{*}$ \\
\hline $\mathrm{Co}(\mathrm{I})+\mathrm{H}^{+} \rightarrow \mathrm{Co}-\mathrm{H}$ & 500 & $2 \mathrm{e} 3$ & 4 \\
\hline $\mathrm{s}+\mathrm{s} \rightarrow \mathrm{H}_{2}$ & 1 & $1 \mathrm{e} 10$ & $1 \mathrm{e} 10$ \\
\hline $\mathrm{Co}-\mathrm{H}+\mathrm{Co}-\mathrm{H} \rightarrow \mathrm{A}+\mathrm{H}_{2}$ & 1000 & $3 \mathrm{e} 5$ & 300 \\
\hline $\mathrm{A} \rightarrow \mathrm{Co}(\mathrm{II})+\mathrm{Co}(\mathrm{II})$ & $4.5 \mathrm{e} 5^{*}$ & $1 \mathrm{e} 10$ & $2.2 \mathrm{e} 4$ \\
\hline
\end{tabular}

* These constants are automatically calculated by the program

\section{Species:}

\begin{tabular}{|c|c|c|c|c|c|c|c|c|}
\hline & $\mathrm{D}\left(\mathrm{cm}^{2} / \mathrm{s}\right)$ & $\mathrm{C}(\mathrm{M})$ & & $\mathrm{D}\left(\mathrm{cm}^{2} / \mathrm{s}\right)$ & $\mathrm{C}(\mathrm{M})$ & & $\mathrm{D}\left(\mathrm{cm}^{2} / \mathrm{s}\right)$ & $\mathrm{C}(\mathrm{M})$ \\
\hline $\mathrm{Co}(\mathrm{III})$ & $1.2 \mathrm{e}-5$ & 0 & $\mathrm{Co}-\mathrm{H}$ & $1.2 \mathrm{e}-5$ & 0 & $\mathrm{~A}$ & $1.2 \mathrm{e}-5$ & 0 \\
\hline $\mathrm{Co}(\mathrm{II})$ & $1.2 \mathrm{e}-5$ & $1.59 \mathrm{e}-4$ & $\mathrm{H}_{2}$ & $1 e-5$ & 0 & & & \\
\hline $\mathrm{Co}(\mathrm{I})$ & $1.2 \mathrm{e}-5$ & 0 & $\mathrm{~s}$ & $1 e-5$ & 0 & & & \\
\hline
\end{tabular}

Acid concentrations, $\mathrm{H}^{+}$represents $\mathrm{TsOH}$

\begin{tabular}{|c|c|c|c|c|c|c|}
\hline & $\mathrm{D}\left(\mathrm{cm}^{2} / \mathrm{s}\right)$ & $\mathrm{C}(\mathrm{M})$ & $\mathrm{C}(\mathrm{M})$ & $\mathrm{C}(\mathrm{M})$ & $\mathrm{C}(\mathrm{M})$ & $\mathrm{C}(\mathrm{M})$ \\
\hline $\mathrm{H}^{+}$ & $1 \mathrm{e}-5$ & 0 & $2.8 \mathrm{e}-3$ & $8.4 \mathrm{e}-3$ & $16.8 \mathrm{e}-3$ & $22.4 \mathrm{e}-3$ \\
\hline
\end{tabular}



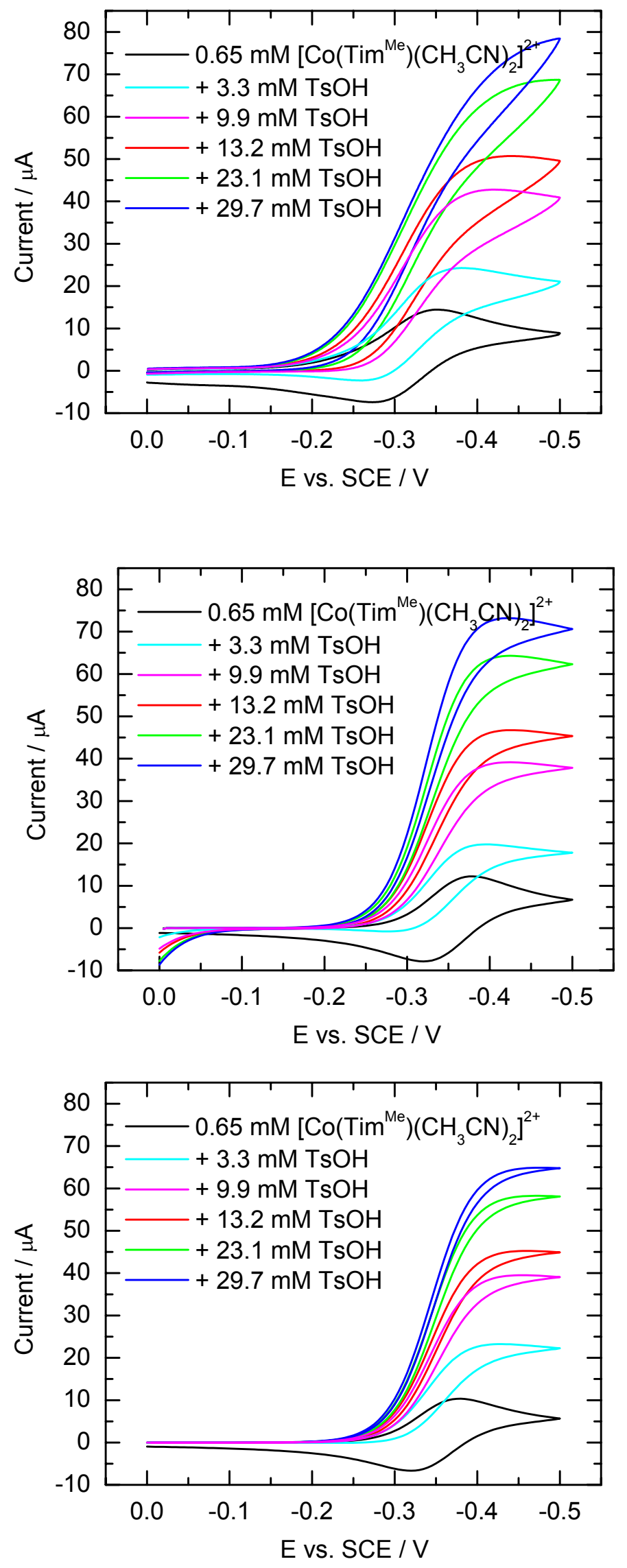
Figure S14. Experimental and simulated spectra of the catalytic waves for $\mathrm{H}_{2}$ evolution catalysis using $0.65 \mathrm{mM}$ of $\mathbf{4 b}$ as the catalyst; top: experimental data; middle: simulation using a monometallic mechanism; bottom: simulation using a bimetallic mechanism. As shown in the figure, the simulations can not distinguish a monometallic mechanism from a bimetallic mechanism.

Specific Parameters for Simulation in Figure S14:

\begin{tabular}{|c|c|c|}
\hline Estart (V) & Erev (V) & Eend (V) \\
\hline 0 & -0.5 & 0 \\
\hline
\end{tabular}

Charge Transfer reactions:

\begin{tabular}{|c|c|c|c|}
\hline Rxn & E (V) & $\alpha$ & Ks (cm/s) \\
\hline $\mathrm{Co}(\mathrm{III})+\mathrm{e}^{-} \rightarrow \mathrm{Co}(\mathrm{II})$ & 0.2 & 0.5 & 10000 \\
\hline $\mathrm{Co}(\mathrm{II})+\mathrm{e}^{-} \rightarrow \mathrm{Co}(\mathrm{II})$ & -0.35 & 0.5 & 10000 \\
\hline $\mathrm{H}^{+}+\mathrm{e}^{-} \rightarrow \mathrm{s}$ & -0.23 & 0.5 & 0 \\
\hline
\end{tabular}

Chemical reactions for monometallic mechanism:

\begin{tabular}{|c|c|c|c|}
\hline Rxn & Keq & kf & $\mathrm{kb}^{*}$ \\
\hline $\mathrm{Co}(\mathrm{I})+\mathrm{H}^{+} \rightarrow \mathrm{Co}-\mathrm{H}$ & 300 & 8000 & 26.6 \\
\hline $\mathrm{s}+\mathrm{s} \rightarrow \mathrm{H}_{2}$ & 1 & $1 \mathrm{e} 10$ & $1 \mathrm{e} 10$ \\
\hline $\mathrm{Co}-\mathrm{H}+\mathrm{H}^{+} \rightarrow \mathrm{Co}(\mathrm{III})+\mathrm{H}_{2}$ & $1.9 \mathrm{e}-8$ & 650 & $4 \mathrm{e} 10$ \\
\hline $\mathrm{Co}(\mathrm{III})+\mathrm{Co}(\mathrm{I}) \rightarrow \mathrm{Co}(\mathrm{II})+\mathrm{Co}(\mathrm{II})$ & $1.9 \mathrm{e} 9 *$ & $1 \mathrm{e} 10$ & 5 \\
\hline
\end{tabular}


Chemical reactions for bimetallic mechanism:

\begin{tabular}{|c|c|c|c|}
\hline Rxn & Keq & kf & $\mathrm{kb}^{*}$ \\
\hline $\mathrm{Co}(\mathrm{I})+\mathrm{H}^{+} \rightarrow \mathrm{Co}-\mathrm{H}$ & 500 & $2 \mathrm{e} 3$ & 4 \\
\hline $\mathrm{s}+\mathrm{s} \rightarrow \mathrm{H}_{2}$ & 1 & $1 \mathrm{e} 10$ & $1 \mathrm{e} 10$ \\
\hline $\mathrm{Co}-\mathrm{H}+\mathrm{Co}-\mathrm{H} \rightarrow \mathrm{A}+\mathrm{H}_{2}$ & 1000 & $3 \mathrm{e} 5$ & 300 \\
\hline $\mathrm{A} \rightarrow \mathrm{Co}(\mathrm{II})+\mathrm{Co}(\mathrm{II})$ & $4.5 \mathrm{e} 5^{*}$ & $1 \mathrm{e} 10$ & $2.2 \mathrm{e} 4$ \\
\hline
\end{tabular}

* These constants are automatically calculated by the program

Species:

\begin{tabular}{|c|c|c|c|c|c|c|c|c|}
\hline & $\mathrm{D}\left(\mathrm{cm}^{2} / \mathrm{s}\right)$ & $\mathrm{C}(\mathrm{M})$ & & $\mathrm{D}\left(\mathrm{cm}^{2} / \mathrm{s}\right)$ & $\mathrm{C}(\mathrm{M})$ & & $\mathrm{D}\left(\mathrm{cm}^{2} / \mathrm{s}\right)$ & $\mathrm{C}(\mathrm{M})$ \\
\hline $\mathrm{Co}(\mathrm{III})$ & $1.2 \mathrm{e}-5$ & 0 & $\mathrm{Co}-\mathrm{H}$ & $1.2 \mathrm{e}-5$ & 0 & $\mathrm{~A}$ & $1.2 \mathrm{e}-5$ & 0 \\
\hline $\mathrm{Co}(\mathrm{II})$ & $1.2 \mathrm{e}-5$ & $1.59 \mathrm{e}-4$ & $\mathrm{H}_{2}$ & $1 \mathrm{e}-5$ & 0 & & & \\
\hline $\mathrm{Co}(\mathrm{I})$ & $1.2 \mathrm{e}-5$ & 0 & $S$ & $1 \mathrm{e}-5$ & 0 & & & \\
\hline
\end{tabular}

Acid concentrations, $\mathrm{H}^{+}$represents $\mathrm{TsOH}$

\begin{tabular}{|l|c|c|c|c|c|c|c|}
\hline & $\mathrm{D}\left(\mathrm{cm}^{2} / \mathrm{s}\right)$ & $\mathrm{C}(\mathrm{M})$ & $\mathrm{C}(\mathrm{M})$ & $\mathrm{C}(\mathrm{M})$ & $\mathrm{C}(\mathrm{M})$ & $\mathrm{C}(\mathrm{M})$ & $\mathrm{C}(\mathrm{M})$ \\
\hline $\mathrm{H}^{+}$ & $1 \mathrm{e}-5$ & 0 & $3.3 \mathrm{e}-3$ & $9.9 \mathrm{e}-3$ & $13.2 \mathrm{e}-3$ & $23.1 \mathrm{e}-3$ & $29.7 \mathrm{e}-3$ \\
\hline
\end{tabular}




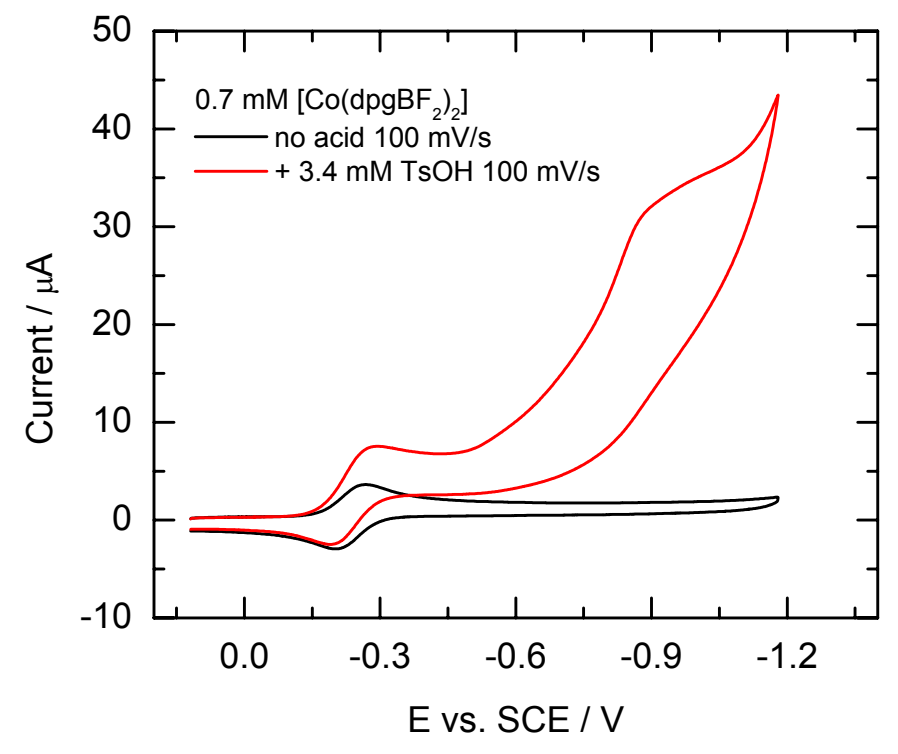

Figure S15. Cyclic voltammogram of $0.7 \mathrm{mM} 2$ in $\mathrm{CH}_{3} \mathrm{CN}$ solution containing $0.1 \mathrm{M}$ $\left[{ }^{\mathrm{n}} \mathrm{Bu}_{4} \mathrm{~N}\right]\left[\mathrm{ClO}_{4}\right]$ in the absence (black) and presence (red) of $3.4 \mathrm{mM} \mathrm{TsOH} \cdot \mathrm{H}_{2} \mathrm{O}$ showing a catalytic wave negative of the $\mathrm{Co}{ }^{\mathrm{II} / \mathrm{I}}$ potential. Scan rate: $100 \mathrm{mV} / \mathrm{s}^{-1}$; Glassy carbon electrode. 


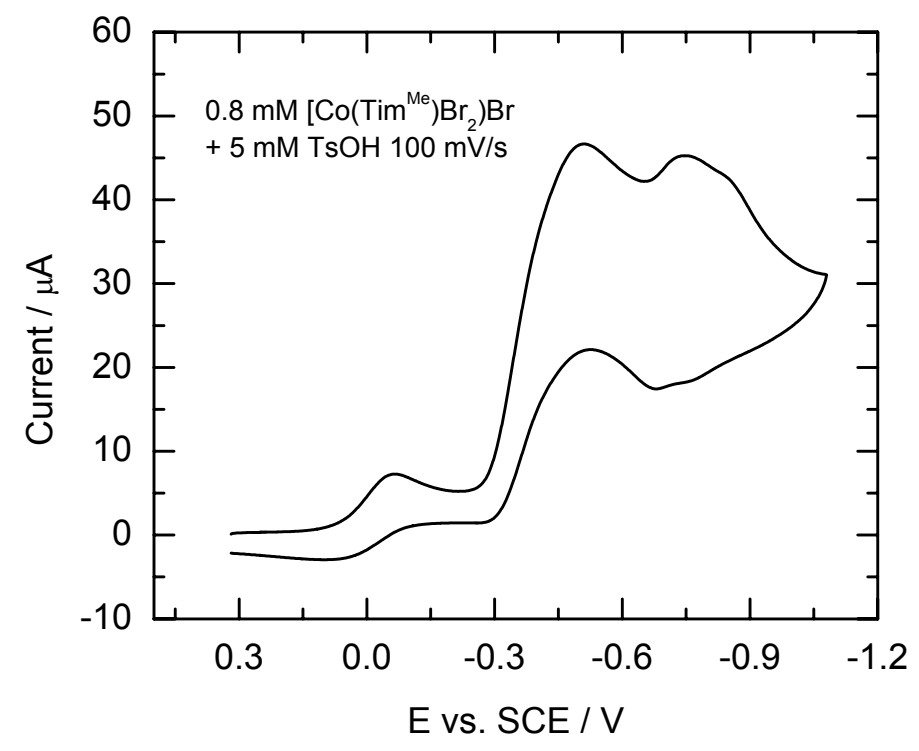

Figure S16. Cyclic voltammogram of $0.8 \mathrm{mM}$ 4a in $\mathrm{CH}_{3} \mathrm{CN}$ solution containing $0.1 \mathrm{M}$ $\left[{ }^{\mathrm{n}} \mathrm{Bu}_{4} \mathrm{~N}\right]\left[\mathrm{ClO}_{4}\right]$ in presence of $5 \mathrm{mM} \mathrm{TsOH} \cdot \mathrm{H}_{2} \mathrm{O}$ showing a redox wave negative of the $\mathrm{Co}^{\mathrm{II} / \mathrm{I}}$ potential. Scan rate: $100 \mathrm{mV} / \mathrm{s}^{-1}$; Glassy carbon electrode. 

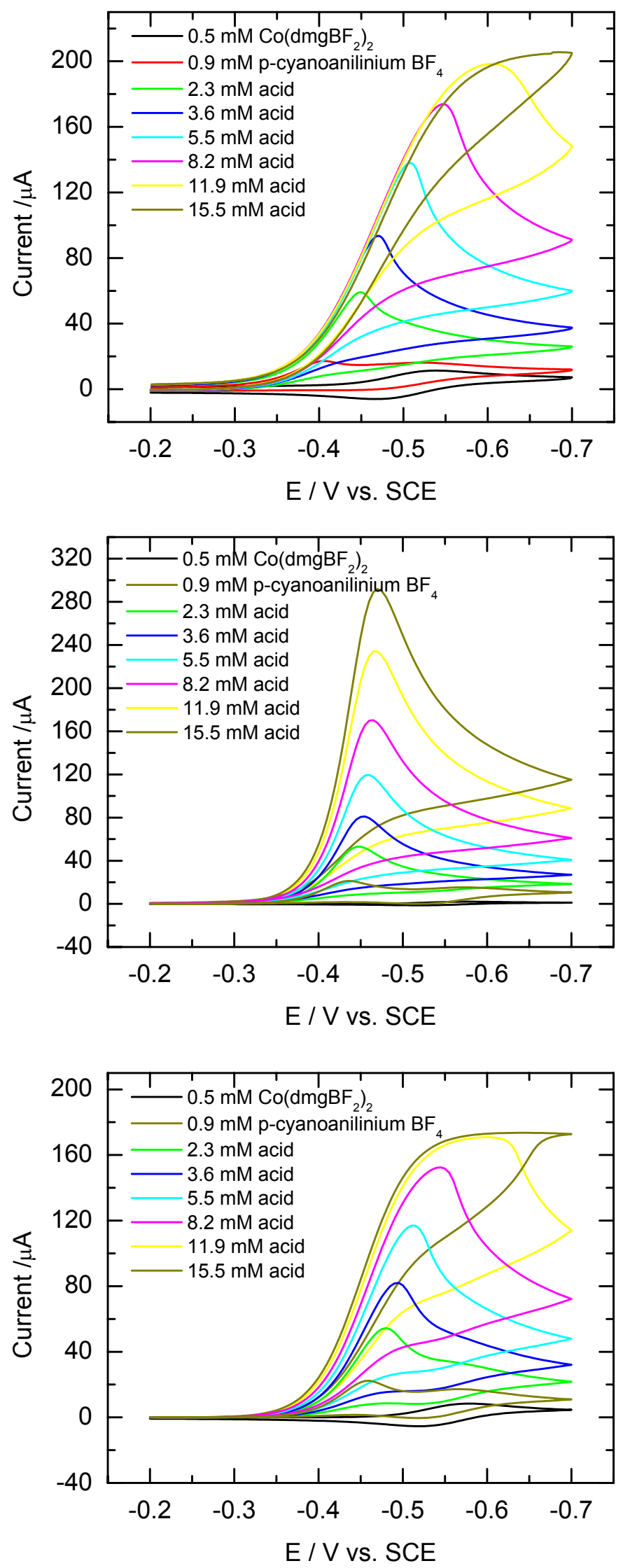
Figure S17. Experimental and simulated spectra of the catalytic waves for $\mathrm{H}_{2}$ evolution catalysis using $0.5 \mathrm{mM}$ of $\mathbf{1}$ as the catalyst and p-cyanoanilinium $\mathrm{BF}_{4}$ as the acid; top: experimental data; middle: simulation using a monometallic mechanism; bottom: simulation using a bimetallic mechanism. As shown in the figure, only simulations assuming a bimetallic mechanism could give rise to satisfactory fits to the experimental data collected under all conditions.

Specific Parameters for Simulation in Figure S17:

\begin{tabular}{|c|c|c|}
\hline Estart (V) & Erev (V) & Eend (V) \\
\hline 0 & -0.5 & 0 \\
\hline
\end{tabular}

\section{Charge Transfer reactions:}

\begin{tabular}{|c|c|c|c|}
\hline Rxn & E (V) & $\alpha$ & $\mathrm{Ks}(\mathrm{cm} / \mathrm{s})$ \\
\hline $\mathrm{Co}(\mathrm{III})+\mathrm{e}^{-} \rightarrow \mathrm{Co}(\mathrm{II})$ & 0.2 & 0.5 & 10000 \\
\hline $\mathrm{Co}(\mathrm{II})+\mathrm{e}^{-} \rightarrow \mathrm{Co}(\mathrm{II})$ & -0.35 & 0.5 & 10000 \\
\hline $\mathrm{H}^{+}+\mathrm{e}^{-} \rightarrow \mathrm{s}$ & -0.20 & 0.5 & 0 \\
\hline
\end{tabular}

Chemical reactions for monometallic mechanism:

\begin{tabular}{|c|c|c|c|}
\hline $\mathrm{Rxn}$ & $\mathrm{Keq}$ & $\mathrm{kf}$ & $\mathrm{kb}$ \\
\hline $\mathrm{Co}(\mathrm{I})+\mathrm{H}^{+} \rightarrow \mathrm{Co}-\mathrm{H}$ & $3 \mathrm{e} 5$ & $7 \mathrm{e} 7$ & 233 \\
\hline $\mathrm{s}+\mathrm{s} \rightarrow \mathrm{H}_{2}$ & 1 & $1 \mathrm{e} 10$ & $1 \mathrm{e} 10$ \\
\hline $\mathrm{Co}-\mathrm{H}+\mathrm{H}^{+} \rightarrow \mathrm{Co}(\mathrm{III})+\mathrm{H}_{2}$ & $4.7 \mathrm{e}-7$ & $1 \mathrm{e} 10$ & $2 \mathrm{e} 16$ \\
\hline $\mathrm{Co}(\mathrm{III})+\mathrm{Co}(\mathrm{I}) \rightarrow \mathrm{Co}(\mathrm{II})+\mathrm{Co}(\mathrm{II})$ & $4.7 \mathrm{e} 12^{*}$ & $1 \mathrm{e} 10$ & 0.002 \\
\hline
\end{tabular}


Chemical reactions for bimetallic mechanism:

\begin{tabular}{|c|c|c|c|}
\hline Rxn & Keq & $\mathrm{kf}$ & $\mathrm{kb}^{*}$ \\
\hline $\mathrm{Co}(\mathrm{I})+\mathrm{H}^{+} \rightarrow \mathrm{Co}-\mathrm{H}$ & $3 \mathrm{e} 5$ & $1 \mathrm{e} 8$ & 333 \\
\hline $\mathrm{s}+\mathrm{s} \rightarrow \mathrm{H}_{2}$ & 1 & $1 \mathrm{e} 10$ & $1 \mathrm{e} 10$ \\
\hline $\mathrm{Co}-\mathrm{H}+\mathrm{Co}-\mathrm{H} \rightarrow \mathrm{A}+\mathrm{H}_{2}$ & 1 & $5 \mathrm{e} 5$ & $5 \mathrm{e} 5$ \\
\hline $\mathrm{A} \rightarrow \mathrm{Co}(\mathrm{II})+\mathrm{Co}(\mathrm{II})$ & $7.5^{*}$ & $1 \mathrm{e} 10$ & $1.3 \mathrm{e} 9$ \\
\hline
\end{tabular}

* These constants are automatically calculated by the program

Species:

\begin{tabular}{|c|c|c|c|c|c|c|c|c|}
\hline & $\mathrm{D}\left(\mathrm{cm}^{2} / \mathrm{s}\right)$ & $\mathrm{C}(\mathrm{M})$ & & $\mathrm{D}\left(\mathrm{cm}^{2} / \mathrm{s}\right)$ & $\mathrm{C}(\mathrm{M})$ & & $\mathrm{D}\left(\mathrm{cm}^{2} / \mathrm{s}\right)$ & $\mathrm{C}(\mathrm{M})$ \\
\hline $\mathrm{Co}(\mathrm{III})$ & $1.2 \mathrm{e}-5$ & 0 & Co-H & $1.2 \mathrm{e}-5$ & 0 & A & $1.2 \mathrm{e}-5$ & 0 \\
\hline $\mathrm{Co}(\mathrm{II})$ & $1.2 \mathrm{e}-5$ & $5 e-4$ & $\mathrm{H}_{2}$ & $1 \mathrm{e}-5$ & 0 & & & \\
\hline $\mathrm{Co}(\mathrm{I})$ & $1.2 \mathrm{e}-5$ & 0 & s & $1 e-5$ & 0 & & & \\
\hline
\end{tabular}

Acid concentrations, $\mathrm{H}^{+}$represents p-cyanoanilinium.

\begin{tabular}{|c|c|c|c|c|c|c|c|c|c|}
\hline & $\mathrm{D}\left(\mathrm{cm}^{2} / \mathrm{s}\right)$ & $\mathrm{C}(\mathrm{M})$ & $\mathrm{C}(\mathrm{M})$ & $\mathrm{C}(\mathrm{M})$ & $\mathrm{C}(\mathrm{M})$ & $\mathrm{C}(\mathrm{M})$ & $\mathrm{C}(\mathrm{M})$ & $\mathrm{C}(\mathrm{M})$ & $\mathrm{C}(\mathrm{M})$ \\
\hline $\mathrm{H}^{+}$ & $1 \mathrm{e}-5$ & 0 & $0.9 \mathrm{e}-3$ & $2.3 \mathrm{e}-3$ & $3.6 \mathrm{e}-3$ & $5.5 \mathrm{e}-3$ & $8.2 \mathrm{e}-3$ & $11.9 \mathrm{e}-3$ & $15.5 \mathrm{e}-3$ \\
\hline
\end{tabular}



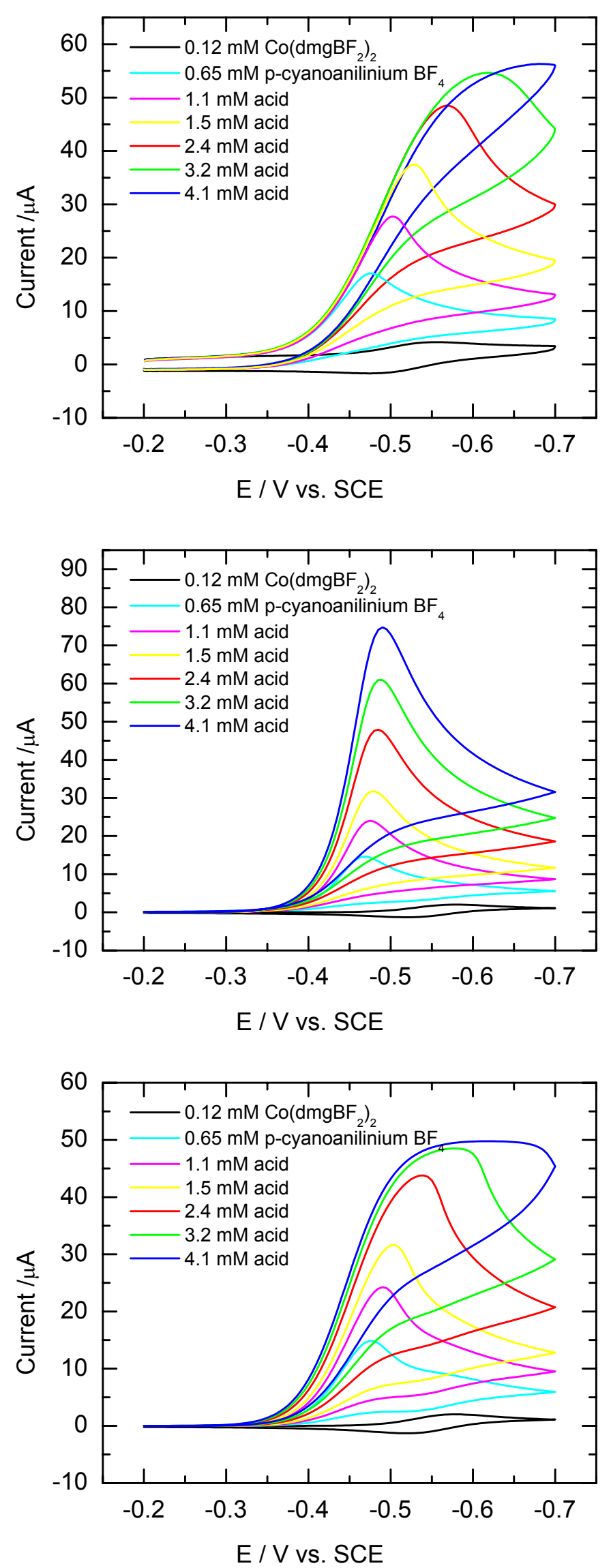
Figure S18. Experimental and simulated spectra of the catalytic waves for $\mathrm{H}_{2}$ evolution catalysis using $0.12 \mathrm{mM}$ of $\mathbf{1}$ as the catalyst and p-cyanoanilinium $\mathrm{BF}_{4}$ as the acid; top: experimental data; middle: simulation using a monometallic mechanism; bottom: simulation using a bimetallic mechanism. As shown in the figure, only simulations assuming a bimetallic mechanism could give rise to satisfactory fits to the experimental data collected under all conditions.

Specific Parameters for Simulation in Figure S18:

\begin{tabular}{|c|c|c|}
\hline Estart (V) & Erev (V) & Eend (V) \\
\hline 0 & -0.5 & 0 \\
\hline
\end{tabular}

\section{Charge Transfer reactions:}

\begin{tabular}{|c|c|c|c|}
\hline Rxn & E (V) & $\alpha$ & $\mathrm{Ks}(\mathrm{cm} / \mathrm{s})$ \\
\hline $\mathrm{Co}(\mathrm{III})+\mathrm{e}^{-} \rightarrow \mathrm{Co}(\mathrm{II})$ & 0.2 & 0.5 & 10000 \\
\hline $\mathrm{Co}(\mathrm{II})+\mathrm{e}^{-} \rightarrow \mathrm{Co}(\mathrm{II})$ & -0.35 & 0.5 & 10000 \\
\hline $\mathrm{H}^{+}+\mathrm{e}^{-} \rightarrow \mathrm{s}$ & -0.20 & 0.5 & 0 \\
\hline
\end{tabular}

\section{Chemical reactions for monometallic mechanism:}

\begin{tabular}{|c|c|c|c|}
\hline $\mathrm{Rxn}$ & $\mathrm{Keq}$ & $\mathrm{kf}$ & $\mathrm{kb}$ \\
\hline $\mathrm{Co}(\mathrm{I})+\mathrm{H}^{+} \rightarrow \mathrm{Co}-\mathrm{H}$ & $3 \mathrm{e} 5$ & $7 \mathrm{e} 7$ & 233 \\
\hline $\mathrm{s}+\mathrm{s} \rightarrow \mathrm{H}_{2}$ & 1 & $1 \mathrm{e} 10$ & $1 \mathrm{e} 10$ \\
\hline $\mathrm{Co}-\mathrm{H}+\mathrm{H}^{+} \rightarrow \mathrm{Co}(\mathrm{III})+\mathrm{H}_{2}$ & $4.7 \mathrm{e}-7$ & $1 \mathrm{e} 10$ & $2 \mathrm{e} 16$ \\
\hline $\mathrm{Co}(\mathrm{III})+\mathrm{Co}(\mathrm{I}) \rightarrow \mathrm{Co}(\mathrm{II})+\mathrm{Co}(\mathrm{II})$ & $4.7 \mathrm{e} 12^{*}$ & $1 \mathrm{e} 10$ & 0.002 \\
\hline
\end{tabular}


Chemical reactions for bimetallic mechanism:

\begin{tabular}{|c|c|c|c|}
\hline Rxn & Keq & $\mathrm{kf}$ & $\mathrm{kb}^{*}$ \\
\hline $\mathrm{Co}(\mathrm{I})+\mathrm{H}^{+} \rightarrow \mathrm{Co}-\mathrm{H}$ & $3 \mathrm{e} 5$ & $1 \mathrm{e} 9$ & 3333 \\
\hline $\mathrm{s}+\mathrm{s} \rightarrow \mathrm{H}_{2}$ & 1 & $1 \mathrm{e} 10$ & $1 \mathrm{e} 10$ \\
\hline $\mathrm{Co}-\mathrm{H}+\mathrm{Co}-\mathrm{H} \rightarrow \mathrm{A}+\mathrm{H}_{2}$ & 1 & $3 \mathrm{e} 6$ & $5 \mathrm{e} 5$ \\
\hline $\mathrm{A} \rightarrow \mathrm{Co}(\mathrm{II})+\mathrm{Co}(\mathrm{II})$ & $7.5^{*}$ & $1 \mathrm{e} 10$ & $1.3 \mathrm{e} 9$ \\
\hline
\end{tabular}

* These constants are automatically calculated by the program

Species:

\begin{tabular}{|c|c|c|c|c|c|c|c|c|}
\hline & $\mathrm{D}\left(\mathrm{cm}^{2} / \mathrm{s}\right)$ & $\mathrm{C}(\mathrm{M})$ & & $\mathrm{D}\left(\mathrm{cm}^{2} / \mathrm{s}\right)$ & $\mathrm{C}(\mathrm{M})$ & & $\mathrm{D}\left(\mathrm{cm}^{2} / \mathrm{s}\right)$ & $\mathrm{C}(\mathrm{M})$ \\
\hline $\mathrm{Co}(\mathrm{III})$ & $1.2 \mathrm{e}-5$ & 0 & $\mathrm{Co}-\mathrm{H}$ & $1.2 \mathrm{e}-5$ & 0 & A & $1.2 \mathrm{e}-5$ & 0 \\
\hline $\mathrm{Co}$ (II) & $1.2 \mathrm{e}-5$ & $1.2 \mathrm{e}-4$ & $\mathrm{H}_{2}$ & $1 e-5$ & 0 & & & \\
\hline $\mathrm{Co}(\mathrm{I})$ & $1.2 \mathrm{e}-5$ & 0 & $\mathrm{~s}$ & $1 e-5$ & 0 & & & \\
\hline
\end{tabular}

Acid concentrations, $\mathrm{H}^{+}$represents p-cyanoanilinium.

\begin{tabular}{|l|c|c|l|l|l|l|l|l|}
\hline & $\mathrm{D}\left(\mathrm{cm}^{2} / \mathrm{s}\right)$ & $\mathrm{C}(\mathrm{M})$ & $\mathrm{C}(\mathrm{M})$ & $\mathrm{C}(\mathrm{M})$ & $\mathrm{C}(\mathrm{M})$ & $\mathrm{C}(\mathrm{M})$ & $\mathrm{C}(\mathrm{M})$ & $\mathrm{C}(\mathrm{M})$ \\
\hline $\mathrm{H}^{+}$ & $1 \mathrm{e}-5$ & 0 & $0.65 \mathrm{e}-3$ & $1.1 \mathrm{e}-3$ & $1.5 \mathrm{e}-3$ & $2.4 \mathrm{e}-3$ & $3.2 \mathrm{e}-3$ & $4.1 \mathrm{e}-3$ \\
\hline
\end{tabular}

